\title{
Diffractive dijet production with a leading proton in $e p$ collisions at HERA
}

\section{The $\mathrm{H} 1$ collaboration}

V. Andreev, ${ }^{21}$ A. Baghdasaryan, ${ }^{33}$ K. Begzsuren, ${ }^{30}$ A. Belousov, ${ }^{21}$ P. Belov, ${ }^{10}$ V. Boudry, ${ }^{24}$ G. Brandt, ${ }^{45}$ M. Brinkmann, ${ }^{10}$ V. Brisson, ${ }^{23}$ D. Britzger, ${ }^{10}$

A. Buniatyan, ${ }^{2}$ A. Bylinkin, ${ }^{20,42}$ L. Bystritskaya, ${ }^{20}$ A.J. Campbell, ${ }^{10}$

K.B. Cantun Avila, ${ }^{19}$ F. Ceccopieri, ${ }^{3}$ K. Cerny, ${ }^{27}$ V. Chekelian, ${ }^{22}$ J.G. Contreras, ${ }^{19}$

J. Cvach, ${ }^{26}$ J.B. Dainton, ${ }^{16}$ K. Daum, ${ }^{32,37}$ C. Diaconu, ${ }^{18}$ M. Dobre, ${ }^{4}$ V. Dodonov, ${ }^{10}$

A. Dossanov, ${ }^{11,22}$ G. Eckerlin, ${ }^{10}$ S. Egli, ${ }^{31}$ E. Elsen, ${ }^{10}$ L. Favart, ${ }^{3}$ A. Fedotov, ${ }^{20}$

J. Feltesse, ${ }^{9}$ J. Ferencei, ${ }^{14}$ M. Fleischer, ${ }^{10}$ A. Fomenko, ${ }^{21}$ E. Gabathuler, ${ }^{16}$ J. Gayler, ${ }^{10}$

S. Ghazaryan, ${ }^{10}$ A. Glazov, ${ }^{10}$ L. Goerlich, ${ }^{6}$ N. Gogitidze, ${ }^{21}$ M. Gouzevitch, ${ }^{10,38}$

C. Grab, ${ }^{35}$ A. Grebenyuk, ${ }^{3}$ T. Greenshaw, ${ }^{16}$ G. Grindhammer, ${ }^{22}$ D. Haidt, ${ }^{10}$

R.C.W. Henderson, ${ }^{15}$ M. Herbst, ${ }^{13}$ J. Hladkỳ, ${ }^{26}$ D. Hoffmann, ${ }^{18}$ R. Horisberger, ${ }^{31}$

T. Hreus, ${ }^{3}$ F. Huber, ${ }^{12}$ M. Jacquet, ${ }^{23}$ X. Janssen, ${ }^{3}$ H. Jung, ${ }^{10,3}$ M. Kapichine, ${ }^{8}$

C. Kiesling, ${ }^{22}$ M. Klein, ${ }^{16}$ C. Kleinwort, ${ }^{10}$ R. Kogler, ${ }^{11}$ P. Kostka, ${ }^{16}$ J. Kretzschmar, ${ }^{16}$

K. Krüger, ${ }^{10}$ M.P.J. Landon, ${ }^{17}$ W. Lange, ${ }^{34}$ P. Laycock, ${ }^{16}$ A. Lebedev, ${ }^{21}$

S. Levonian, ${ }^{10}$ K. Lipka, ${ }^{10,41}$ B. List, ${ }^{10}$ J. List, ${ }^{10}$ B. Lobodzinski, ${ }^{22}$ E. Malinovski, ${ }^{21}$

H.-U. Martyn, ${ }^{1}$ S.J. Maxfield, ${ }^{16}$ A. Mehta, ${ }^{16}$ A.B. Meyer, ${ }^{10}$ H. Meyer, ${ }^{32}$ J. Meyer, ${ }^{10}$

S. Mikocki, ${ }^{6}$ A. Morozov, ${ }^{8}$ K. Müller, ${ }^{36}$ Th. Naumann, ${ }^{34}$ P.R. Newman, ${ }^{2}$

C. Niebuhr, ${ }^{10}$ G. Nowak, ${ }^{6}$ J.E. Olsson, ${ }^{10}$ D. Ozerov, ${ }^{10}$ P. Pahl, ${ }^{10}$ C. Pascaud, ${ }^{23}$

G.D. Patel, ${ }^{16}$ E. Perez, ${ }^{9,39}$ A. Petrukhin, ${ }^{10}$ I. Picuric, ${ }^{25}$ H. Pirumov, ${ }^{10}$ D. Pitzl, ${ }^{10}$

R. Plačakytè, ${ }^{10,41}$ B. Pokorny, ${ }^{27}$ R. Polifka, ${ }^{27,43}$ V. Radescu, ${ }^{10,41}$ N. Raicevic, ${ }^{25}$

T. Ravdandorj, ${ }^{30}$ P. Reimer, ${ }^{26}$ E. Rizvi, ${ }^{17}$ P. Robmann, ${ }^{36}$ R. Roosen, ${ }^{3}$ A. Rostovtsev, ${ }^{20}$

M. Rotaru, ${ }^{4}$ S. Rusakov, ${ }^{21}$ D. Šálek, ${ }^{27}$ D.P.C. Sankey, ${ }^{5}$ M. Sauter, ${ }^{12}$ E. Sauvan, ${ }^{18,44}$

S. Schmitt, ${ }^{10}$ L. Schoeffel, ${ }^{9}$ A. Schöning, ${ }^{12}$ H.-C. Schultz-Coulon, ${ }^{13}$ F. Sefkow, ${ }^{10}$

S. Shushkevich, ${ }^{10}$ Y. Soloviev, ${ }^{10,21}$ P. Sopicki, ${ }^{6}$ D. South, ${ }^{10}$ V. Spaskov, ${ }^{8}$ A. Specka, ${ }^{24}$

M. Steder, ${ }^{10}$ B. Stella, ${ }^{28}$ U. Straumann, ${ }^{36}$ T. Sykora, ${ }^{3,27}$ P.D. Thompson, ${ }^{2}$

D. Traynor, ${ }^{17}$ P. Truöl, ${ }^{36}$ I. Tsakov, ${ }^{29}$ B. Tseepeldorj, ${ }^{30,40}$ J. Turnau, ${ }^{6}$ A. Valkárová, ${ }^{27}$

C. Vallée, ${ }^{18}$ P. Van Mechelen, ${ }^{3}$ Y. Vazdik, ${ }^{21}$ D. Wegener, ${ }^{7}$ E. Wünsch, ${ }^{10}$ J. Žáček, ${ }^{27}$

Z. Zhang, ${ }^{23}$ R. Žlebčík, ${ }^{27}$ H. Zohrabyan ${ }^{33}$ and F. Zomer ${ }^{23}$

${ }^{1}$ I. Physikalisches Institut der RWTH, Aachen, Germany 
${ }^{2}$ School of Physics and Astronomy, University of Birmingham, Birmingham, U.K. ${ }^{\mathrm{a}}$

${ }^{3}$ Inter-University Institute for High Energies ULB-VUB, Brussels and Universiteit Antwerpen, Antwerpen, Belgium ${ }^{\mathrm{b}}$

${ }^{4}$ National Institute for Physics and Nuclear Engineering (NIPNE), Bucharest, Romania ${ }^{\mathrm{c}}$

${ }^{5}$ STFC, Rutherford Appleton Laboratory, Didcot, Oxfordshire, U.K. ${ }^{a}$

${ }^{6}$ Institute for Nuclear Physics, Cracow, Poland ${ }^{\mathrm{d}}$

${ }^{7}$ Institut für Physik, TU Dortmund, Dortmund, Germany ${ }^{\mathrm{e}}$

${ }^{8}$ Joint Institute for Nuclear Research, Dubna, Russia

${ }^{9} C E A, D S M / I r f u, C E-S a c l a y$, Gif-sur-Yvette, France

${ }^{10}$ DESY, Hamburg, Germany

${ }^{11}$ Institut für Experimentalphysik, Universität Hamburg, Hamburg, Germany ${ }^{e}$

${ }^{12}$ Physikalisches Institut, Universität Heidelberg, Heidelberg, Germany ${ }^{e}$

${ }^{13}$ Kirchhoff-Institut für Physik, Universität Heidelberg, Heidelberg, Germany ${ }^{e}$

${ }^{14}$ Institute of Experimental Physics, Slovak Academy of Sciences, Košice, Slovak Republic ${ }^{\mathrm{f}}$

${ }^{15}$ Department of Physics, University of Lancaster, Lancaster, U.K. ${ }^{a}$

${ }^{16}$ Department of Physics, University of Liverpool, Liverpool, U.K. ${ }^{a}$

${ }^{17}$ School of Physics and Astronomy, Queen Mary, University of London, London, U.K. ${ }^{a}$

${ }^{18}$ CPPM, Aix-Marseille Univ, CNRS/IN2P3, 13288 Marseille, France

${ }^{19}$ Departamento de Fisica Aplicada, CINVESTAV, Mérida, Yucatán, México ${ }^{\circ}$

${ }^{20}$ Institute for Theoretical and Experimental Physics, Moscow, Russia ${ }^{\mathrm{h}}$

${ }^{21}$ Lebedev Physical Institute, Moscow, Russia

${ }^{22}$ Max-Planck-Institut für Physik, München, Germany

${ }^{23}$ LAL, Université Paris-Sud, CNRS/IN2P3, Orsay, France

${ }^{24}$ LLR, Ecole Polytechnique, CNRS/IN2P3, Palaiseau, France

${ }^{25}$ Faculty of Science, University of Montenegro, Podgorica, Montenegro ${ }^{\mathrm{i}}$

${ }^{26}$ Institute of Physics, Academy of Sciences of the Czech Republic, Praha, Czech Republic ${ }^{\mathrm{j}}$

${ }^{27}$ Faculty of Mathematics and Physics, Charles University, Praha, Czech Republic ${ }^{j}$

${ }^{28}$ Dipartimento di Fisica Università di Roma Tre and INFN Roma 3, Roma, Italy

${ }^{29}$ Institute for Nuclear Research and Nuclear Energy, Sofia, Bulgaria

${ }^{30}$ Institute of Physics and Technology of the Mongolian Academy of Sciences, Ulaanbaatar, Mongolia

${ }^{31}$ Paul Scherrer Institut, Villigen, Switzerland

${ }^{32}$ Fachbereich C, Universität Wuppertal, Wuppertal, Germany

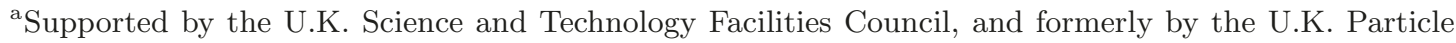
Physics and Astronomy Research Council.

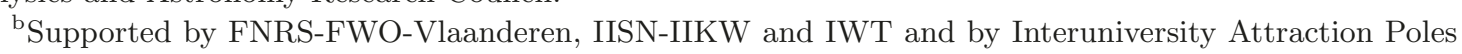
Programme, Belgian Science Policy.

${ }^{\mathrm{c}}$ Supported by the Romanian National Authority for Scientific Research under the contract PN 09370101.

${ }^{\mathrm{d}}$ Partially Supported by Polish Ministry of Science and Higher Education, grant DPN/N168/DESY/2009.

e Supported by the Bundesministerium für Bildung und Forschung, FRG, under contract numbers 05H09GUF, 05H09VHC, 05H09VHF, 05H16PEA.

${ }^{\mathrm{f}}$ Supported by VEGA SR grant no. 2/7062/27.

'Supported by CONACYT, México, grant 48778-F.

${ }^{\mathrm{h}}$ Russian Foundation for Basic Research (RFBR), grant no 1329.2008.2 and Rosatom.

${ }^{i}$ Partially Supported by Ministry of Science of Montenegro, no. 05-1/3-3352.

${ }^{\mathrm{j}}$ Supported by the Ministry of Education of the Czech Republic under the project INGO-LG14033.
} 
${ }^{33}$ Yerevan Physics Institute, Yerevan, Armenia

${ }^{34}$ DESY, Zeuthen, Germany

${ }^{35}$ Institut für Teilchenphysik, ETH, Zürich, Switzerland ${ }^{k}$

${ }^{36}$ Physik-Institut der Universität Zürich, Zürich, Switzerland ${ }^{k}$

${ }^{37}$ Also at Rechenzentrum, Universität Wuppertal, Wuppertal, Germany

${ }^{38}$ Also at IPNL, Université Claude Bernard Lyon 1, CNRS/IN2P3, Villeurbanne, France

${ }^{39}$ Also at CERN, Geneva, Switzerland

${ }^{40}$ Also at Ulaanbaatar University, Ulaanbaatar, Mongolia

${ }^{41}$ Supported by the Initiative and Networking Fund of the Helmholtz Association (HGF) under the contract $V H-N G-401$ and S0-072

${ }^{42}$ Also at Moscow Institute of Physics and Technology, Moscow, Russia

${ }^{43}$ Also at Department of Physics, University of Toronto, Toronto, Ontario, Canada M5S $1 \mathrm{A7}$

${ }^{44}$ Also at LAPP, Université de Savoie, CNRS/IN2P3, Annecy-le-Vieux, France

${ }^{45}$ Department of Physics, Oxford University, Oxford, U.K. ${ }^{a}$

E-mail: daum@mail.desy.de

ABstract: The cross section of the diffractive process $e^{+} p \rightarrow e^{+} X p$ is measured at a centre-of-mass energy of $318 \mathrm{GeV}$, where the system $X$ contains at least two jets and the leading final state proton $p$ is detected in the H1 Very Forward Proton Spectrometer. The measurement is performed in photoproduction with photon virtualities $Q^{2}<2 \mathrm{GeV}^{2}$ and in deep-inelastic scattering with $4 \mathrm{GeV}^{2}<Q^{2}<80 \mathrm{GeV}^{2}$. The results are compared to nextto-leading order QCD calculations based on diffractive parton distribution functions as extracted from measurements of inclusive cross sections in diffractive deep-inelastic scattering.

KEYWORDS: Lepton-Nucleon Scattering, Jets, QCD, Diffraction

ARXIV EPRINT: 1502.01683

\footnotetext{
${ }^{\mathrm{k}}$ Supported by the Swiss National Science Foundation.
} 


\section{Contents}

1 Introduction $\quad 1$

2 Kinematics 3

3 Factorisation in diffractive dijet production 3

$4 \quad$ NLO QCD calculations 5

5 Experimental procedure $\quad 5$

5.1 The H1 detector 5

5.2 Very Forward Proton Spectrometer 6

$\begin{array}{lll}5.3 \text { Kinematic reconstruction } & 6\end{array}$

$\begin{array}{llr}5.3 .1 & \text { DIS } & 8 \\ 5.3 .2 & \text { Photoprodyction }\end{array}$

$\begin{array}{lll}\text { 5.3.2 Photoproduction } & 8\end{array}$

5.4 Event selection 8

5.5 Monte Carlo simulations 9

5.5.1 Correction to the data 9

$\begin{array}{ll}\text { 5.5.2 Correction to theoretical models } & 10\end{array}$

$\begin{array}{lll}5.6 & \text { Cross section measurement } & 11\end{array}$

$\begin{array}{lll}\text { 5.7 Systematic uncertainties of the measured cross section } & 12\end{array}$

6 Results $\quad 14$

6.1 Integrated photoproduction and DIS cross sections 14

$\begin{array}{lll}6.2 & \text { Diffractive dijet production in DIS } & 14\end{array}$

$\begin{array}{lll}6.3 & \text { Diffractive dijet production in photoproduction } & 18\end{array}$

6.4 Comparison of dijet cross sections in diffractive photoproduction and DIS 19

$\begin{array}{lll}7 & \text { Summary } & 27\end{array}$

\section{Introduction}

Diffractive processes, $e p \rightarrow e X Y$, where the systems $X$ and $Y$ are separated in rapidity, have been studied extensively at the electron-proton collider HERA. In diffractive processes the interacting hadrons remain intact or dissociate into low mass hadronic systems via an exchange which has vacuum quantum numbers, often referred to as a pomeron $(\mathbb{P})$. Experimentally, diffractive events may be selected either by the presence of a large rapidity gap (LRG) in the rapidity distribution of the outgoing hadrons or by detecting a leading proton in the final state. The H1 experiment was equipped with two dedicated detectors, 
the Forward Proton Spectrometer (FPS) [1] and the Very Forward Proton Spectrometer (VFPS) [2] to detect the leading protons.

In the framework of the collinear factorisation theorem [3] diffractive parton distribution functions (DPDFs) may be defined. The factorisation theorem predicts that the cross section can be expressed as the convolution of non-perturbative DPDFs and partonic cross sections of the hard sub-process, calculable within perturbative Quantum Chromodynamics (QCD). The DPDFs have properties similar to the parton distribution functions of the proton, but with the constraint of a leading proton or its low mass excitations being present in the final state.

DPDFs were obtained at HERA from inclusive diffractive deep-inelastic scattering (DDIS) data $[4,5]$. Given the DPDFs, perturbative QCD calculations are expected to be applicable to other processes such as jet and heavy quark production in DDIS at HERA [611]. Indeed, next-to-leading order (NLO) QCD predictions using DPDFs describe these measurements well.

In diffractive hadron-hadron interactions however, the production of jets is found to be suppressed by about one order of magnitude $[12,13]$, as compared to predictions based on HERA DPDFs. This "factorisation breaking" may be explained e.g. by soft interactions or multi-pomeron exchanges between the hadrons and/or rescattering phenomena which destroy the diffractive event signature [14-16].

The issues of DPDF applicability and factorisation breaking can also be studied in hard diffractive photoproduction $(\gamma p)$, where the virtuality of the exchanged photon $Q^{2}$ is close to zero. In the photoproduction regime, within the leading order approach, the small photon virtuality allows for partonic fluctuations $\gamma \rightarrow q \bar{q}$ that last long enough to interact with the partons in the proton. In this regime the photon can be treated as a quasi-real target and therefore exhibits hadronic structure.

Diffractive photoproduction of dijets in $e p$ collisions at HERA have been measured by H1 [17, 18] and ZEUS [19]. In each of these measurements diffractive events are selected by requiring a large rapidity gap. Different ratios of data to the NLO QCD prediction have been reported by $\mathrm{H} 1$ and ZEUS: while $\mathrm{H} 1$ reported their data to be suppressed by a factor of 0.6 with respect to the NLO QCD predictions [17, 18], the ZEUS data are compatible with the theoretical expectations [20]. Various mechanisms of suppressing diffractive dijet photoproduction have been proposed [21, 22].

Enhanced sensitivity to the differences between theory and data may be achieved by calculating the double ratio of the ratio of data to predictions of diffractive dijet photoproduction to the corresponding ratio in DDIS [18]. In this way several experimental systematic uncertainties cancel and theoretical uncertainties can be reduced.

In the present paper new measurements of diffractive dijet cross sections in DIS and photoproduction are presented. The data were collected in the years 2006 and 2007 with a total integrated luminosity of $30 \mathrm{pb}^{-1}$ for diffractive photoproduction and $50 \mathrm{pb}^{-1}$ for diffractive DIS. For the identification of diffractive events a proton detected in the VFPS is required. The results are compared to NLO QCD calculations. 


\section{Kinematics}

Figures 1 (a) and (b) show leading order diagrams of direct and resolved diffractive dijet production in $e p$ interactions. The relative contributions of these two components depend on the virtuality of the exchanged photon such that at high virtualities the direct process is dominating. The incoming (scattered) positron four-momentum is denoted as $k\left(k^{\prime}\right)$, the four-momentum of the virtual photon emitted from the positron as $q=k-k^{\prime}$. The four-momentum of the incoming (outgoing) proton is $P\left(P^{\prime}\right)$. The kinematics of the ep scattering process can be described by

$$
s=(k+P)^{2}, \quad Q^{2}=-q^{2}, \quad y=\frac{P \cdot q}{P \cdot k},
$$

where $s$ is the square of the centre-of-mass energy of the collision, $Q^{2}$ is the photon virtuality and $y$ the inelasticity of the process. With $P_{X}$ being the four-momentum of the hadronic final state excluding the leading proton (see figure 1), the inclusive diffractive kinematics is described by the additional variables

$$
M_{X}^{2}=P_{X}^{2}, \quad x_{\mathbb{P}}=\frac{q \cdot\left(P-P^{\prime}\right)}{q \cdot P}, \quad t=\left(P-P^{\prime}\right)^{2},
$$

where $M_{X}$ is the invariant mass of system $X, x_{\mathbb{P}}$ corresponds to the longitudinal momentum fraction lost by the incoming proton and $t$ is the four-momentum transfer squared at the proton vertex.

For diffractive dijet production additional invariants are introduced. With denoting the four-momenta entering the hard sub-process from the photon and from the pomeron side as $u$ and $v$, the longitudinal fractions of the photon and of the pomeron momentum entering the hard sub-process, $x_{\gamma}$ and $z_{\mathbb{P}}$, are defined as

$$
x_{\gamma}=\frac{P \cdot u}{P \cdot q} \quad \text { and } \quad z_{\mathbb{P}}=\frac{q \cdot v}{q \cdot\left(P-P^{\prime}\right)},
$$

respectively.

In leading order, the invariant mass of the dijet system $M_{12}$ is equal to the centre-ofmass energy of the hard sub-process

$$
M_{12}^{2}=(u+v)^{2}
$$

\section{Factorisation in diffractive dijet production}

In the QCD factorisation approach the diffractive dijet cross section is given by the convolution of partonic cross sections $\mathrm{d} \hat{\sigma}$ with diffractive parton distributions $f_{i / p}^{D}$ :

$$
\begin{aligned}
& \mathrm{d} \sigma\left(e p \rightarrow e+2 \text { jets }+X^{\prime}+p\right)=\sum_{i} \int \mathrm{d} t \int \mathrm{d} x_{\mathbb{P}} \int \mathrm{d} z_{\mathbb{P}} \\
& \mathrm{d} \hat{\sigma}_{e i \rightarrow 2 \text { jets }}\left(\hat{s}, \mu_{R}^{2}, \mu_{F}^{2}\right) \times f_{i / p}^{D}\left(z_{\mathbb{P}}, \mu_{F}^{2}, x_{\mathbb{P}}, t\right) .
\end{aligned}
$$




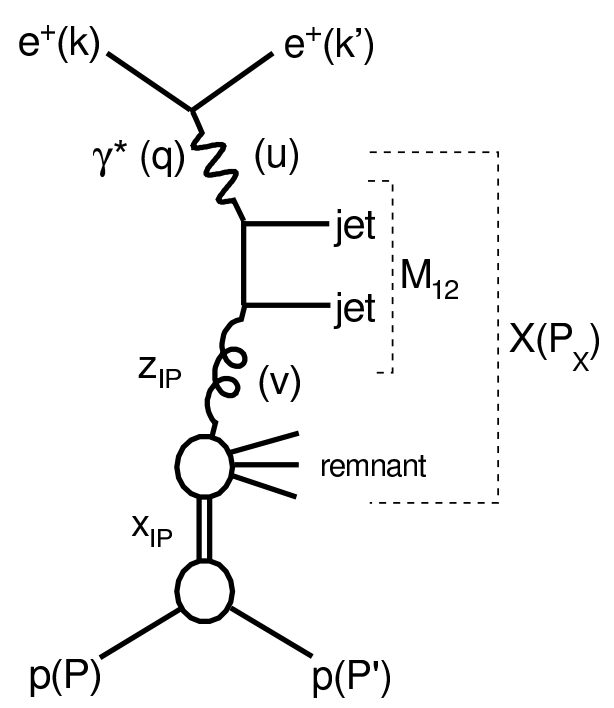

(a)

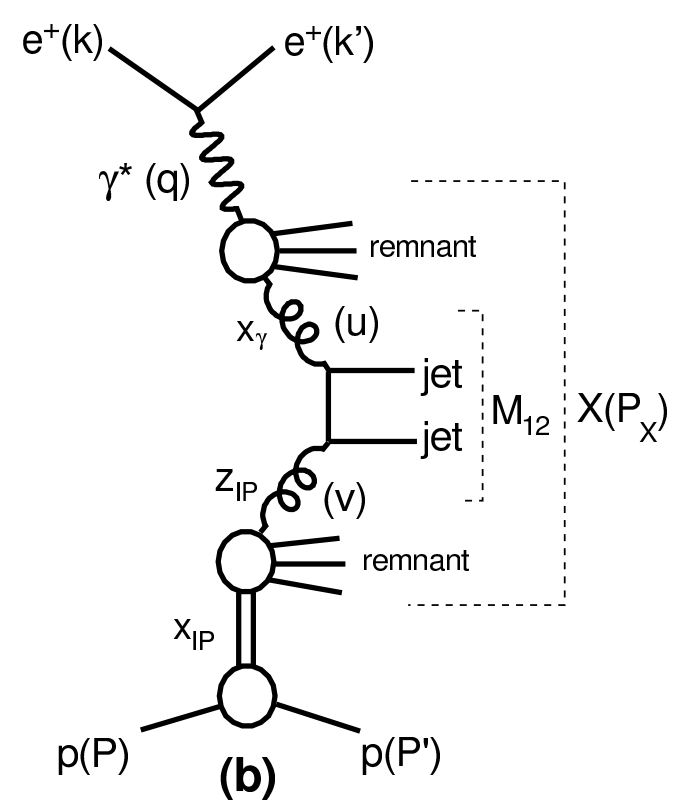

(b)

Figure 1. Leading order diagrams of the direct a) and resolved b) diffractive dijet production.

Here, the hadronic system $X^{\prime}$ corresponds to what remains of the system $X$ after removing the two jets. The integrals extend over the accepted phase space. The sum runs over all partons $i$ contributing to the cross section, $\hat{s} \sim x_{\mathbb{P}} z_{\mathbb{P}} y s-Q^{2}$ is the sub-process invariant energy squared and $\mu_{F}$ and $\mu_{R}$ denote the factorisation and renormalisation scales, respectively.

In the photoproduction region the exchanged photon may dissociate into a low mass non-perturbative hadronic system due to its low virtuality (figure 1b) and a photon parton distribution function $(\gamma \mathrm{PDF})$ is introduced. The cross section for this resolved photon process is given by

$$
\begin{aligned}
& \mathrm{d} \sigma\left(e p \rightarrow e+2 \text { jets }+X^{\prime}+p\right)=\sum_{i, j} \int \mathrm{d} t \int \mathrm{d} x_{\mathbb{P}} \int \mathrm{d} z_{\mathbb{P}} \\
& \int \mathrm{d} y f_{\gamma / e}(y) \int \mathrm{d} x_{\gamma} f_{j / \gamma}\left(x_{\gamma}, \mu_{F}^{2}\right) \times \mathrm{d} \hat{\sigma}_{i j \rightarrow 2 \text { jets }}\left(\hat{s}, \mu_{R}^{2}, \mu_{F}^{2}\right) \times f_{i / p}^{D}\left(z_{\mathbb{P}}, \mu_{F}^{2}, x_{\mathbb{P}}, t\right),
\end{aligned}
$$

where $f_{\gamma / e}$ is the Weizsäcker-Williams equivalent photon flux $[23,24]$ integrated over the measured $Q^{2}$ range and $f_{j / \gamma}$ are the parton distribution functions in the photon ( $\gamma$-PDF). In this case, the centre-of-mass energy of the hard subprocess is approximated by $\hat{s} \sim$ $x_{\gamma} x_{\mathbb{P}} z_{\mathbb{P}} y s$. As default, the GRV [25] $\gamma$-PDFs are used to describe the structure of resolved photons. The AFG [26] $\gamma$-PDF set is also studied.

For the diffractive proton parton densities, the H12006 Fit-B DPDF set [4] is used. This parametrisation was obtained from a QCD fit in NLO accuracy to inclusive DDIS data. In this fit a proton vertex factorisation [27] is assumed in which the $x_{\mathbb{P}}$ and $t$ dependencies of the DPDFs factorise from the dependencies on $\mu_{F}$ and $z_{\mathbb{P}}$ such that

$$
f_{i / p}^{D}\left(z_{\mathbb{P}}, \mu_{F}^{2}, x_{\mathbb{P}}, t\right)=f_{\mathbb{P} / p}\left(x_{\mathbb{P}}, t\right) f_{i / \mathbb{P}}\left(z_{\mathbb{P}}, \mu_{F}^{2}\right)+n_{\mathbb{R}} f_{\mathbb{R} / p}\left(x_{\mathbb{P}}, t\right) f_{i / \mathbb{R}}\left(z_{\mathbb{P}}, \mu_{F}^{2}\right) .
$$


The pomeron flux factor $f_{\mathbb{P} / p}\left(x_{\mathbb{P}}, t\right)$ was parametrised in [4] as suggested by Regge models [28]. For $x_{\mathbb{P}} \gg 0.01$ a small additional contribution from sub-leading reggeon $(\mathbb{R})$ exchange described by the second term in (3.3) was taken into account, where $n_{\mathbb{R}} \sim 10^{-3}$ is the normalisation factor of the reggeon contribution [4].

\section{NLO QCD calculations}

Theoretical calculations of dijet production in next-to-leading order were performed in the $\gamma p$ regime using the the FKS program [29] and in DIS using NLOJET $++[30,31]$. Both programs were adapted [18] for hard diffraction. The NLO calculations for photoproduction are consistent with calculations performed by Klasen and Kramer [32-34]. Similarly, the DDIS predictions were checked using the independent package DISENT NLO [35].

The NLO calculations are performed with the number of flavours fixed to 5 and the QCD scale parameter set to $\Lambda_{5}=0.228 \mathrm{GeV}$, corresponding to a 2-loop $\alpha_{S}\left(M_{Z}\right)$ of 0.118 . The renormalisation and factorisation scales are set to be equal and are calculated from the average jet transverse energy $\left\langle E_{T}^{* \text { jet }}\right\rangle=\left(E_{T}^{* \text { jet1 }}+E_{T}^{* \text { jet2 }}\right) / 2$ and the momentum transfer $Q^{2}$ as $\mu_{R}^{2}=\mu_{F}^{2}=\left\langle E_{T}^{* j e t}\right\rangle^{2}+Q^{2}$. For photoproduction, $Q^{2}$ is set to zero. The sensitivity of the NLO predictions to the scale choice is studied by varying the scale up and down by a factor of two. An alternative definition of the scale $\mu_{R}^{2}=\mu_{F}^{2}=\left(E_{T}^{* \text { jet } 1}\right)^{2}+Q^{2} / 4$, based on the leading jet transverse energy $E_{T}^{* j e t 1}$, is also studied.

\section{Experimental procedure}

\subsection{The $\mathrm{H} 1$ detector}

A detailed description of the $\mathrm{H} 1$ detector can be found elsewhere [36-38]. Here only the detector components most relevant to the present analysis are briefly described. A righthanded coordinate system is employed with the origin at the nominal $e p$ interaction point and with the positive $z$-axis pointing in the proton beam direction. The $x$-axis is pointing along the horizontal direction to the centre of the HERA ring. The pseudorapidity $\eta=$ $-\ln \tan \frac{\theta}{2}$ is calculated using the polar angle $\theta$ measured with respect to the proton beam direction.

The interaction point is surrounded by the central tracking detector (CTD), which consists of a set of concentric drift chambers supplemented by silicon detectors [39] located inside the drift chambers. Charged particle trajectories are bent by a $1.15 \mathrm{~T}$ homogeneous solenoidal magnetic field. The region in pseudorapidity covered by the CTD is $-2.0<$ $\eta<2.0$ and the transverse momentum resolution is $\sigma\left(p_{T}\right) / p_{T} \simeq 0.002 p_{T} / \mathrm{GeV} \oplus 0.015$. A multi-wire proportional chamber at inner radii (CIP) is mainly used for triggering [40]. The forward tracking detector supplements the CTD track reconstructions in the region $7^{\circ}<\theta<25^{\circ}$.

Scattered positrons in the rapidity range $-4<\eta<-1.4$ are measured in a lead / scintillating-fibre calorimeter, the SpaCal [38], with energy resolution $7 \% / \sqrt{E / \mathrm{GeV}} \oplus 1 \%$.

The central and forward tracking detectors are surrounded by a finely segmented Liquid Argon (LAr) calorimeter [41] situated inside the solenoidal magnet and covering the 
pseudorapidity region $-1.5<\eta<3.4$. Its resolution was measured in test beams [42, 43] and is $11 \% / \sqrt{E / \mathrm{GeV}} \oplus 1 \%$ and $50 \% / \sqrt{E / \mathrm{GeV}} \oplus 2 \%$ for electromagnetic and hadronic showers, respectively. The hadronic energy scale is known within $2 \%$ for this analysis [44].

The $e p$ luminosity is determined online by measuring the event rate of the Bethe-Heitler bremsstrahlung process, $e p \rightarrow e p \gamma$, where the photon is detected in a calorimeter located close to the beam pipe at $z=-103 \mathrm{~m}$ [36]. The overall integrated luminosity normalisation is determined using a precision measurement of the QED Compton process [45].

\subsection{Very Forward Proton Spectrometer}

The Very Forward Proton Spectrometer (VFPS) consists of two Roman pots located $218 \mathrm{~m}$ and $222 \mathrm{~m}$ from the interaction point in the forward direction. It allows for a measurement of protons with energies between 895 and $912 \mathrm{GeV}\left(0.008<x_{\mathbb{P}}<0.028\right)$ and with transverse momenta up to about $0.8 \mathrm{GeV}\left(|t|<0.6 \mathrm{GeV}^{2}\right)[2]$.

The VFPS complements the Forward Proton Spectrometer (FPS) [1]. The FPS has a wider acceptance in scattered proton energy $\left(x_{\mathbb{P}}<0.1\right)$ but has only limited geometrical acceptance in the azimuthal angle of the scattered proton (figure 2). In particular at small $|t|<0.2 \mathrm{GeV}^{2}$, the VFPS acceptance is much better than for the FPS. More than $70 \%$ of the diffractive events have $|t|$ smaller than $0.2 \mathrm{GeV}^{2}$.

The Roman pots, which are moved close to the beam as soon as the beam conditions are sufficiently stable, are equipped with detectors made of several layers of scintillating fibers with photomultiplier readout. The sensitive detector areas are covered by scintillator tiles, the signals of which are used as a trigger. The VFPS has high track efficiency $(\sim 96 \%)$ and low background contamination $(\sim 1 \%)$.

\subsection{Kinematic reconstruction}

The observable $x_{\mathbb{P}}$ is reconstructed by the VFPS from the relative distance and angle between the track reconstructed between the two stations and the beam and can be expressed as

$$
x_{\mathbb{P}}=1-\frac{E_{p}^{\prime}}{E_{p}},
$$

where $E_{p}^{\prime}$ is the energy of the leading proton in the VFPS and $E_{p}$ is the proton beam energy.

The quality of the reconstruction of $x_{\mathbb{P}}^{\mathrm{VFPS}}$ was checked using an event sample of elastically produced $\rho$ mesons, $e p \rightarrow e \rho p$. The $x_{\mathbb{P}}^{\rho}$ variable reconstructed from the $\rho$ decay tracks detected in the CTD is compared to $x_{\mathbb{P}}^{\mathrm{VFPS}}$ determined by the VFPS stations. The resulting $x_{\mathbb{P}}^{\mathrm{VFPS}}-x_{\mathbb{P}}^{\rho}$ distributions are found to be in good agreement with Monte Carlo simulations [46]. The resolution of $x_{\mathbb{P}}^{\mathrm{VFPS}}$ was determined to be equal to 0.0022 [2].

The invariant mass $M_{X}$ of the system $X$ is calculated from all hadronic objects in the main $\mathrm{H} 1$ detector:

$$
M_{X}^{2}=\left(\sum_{i \in X} E_{i}\right)^{2}-\left(\sum_{i \in X} \vec{P}_{i}\right)^{2} .
$$




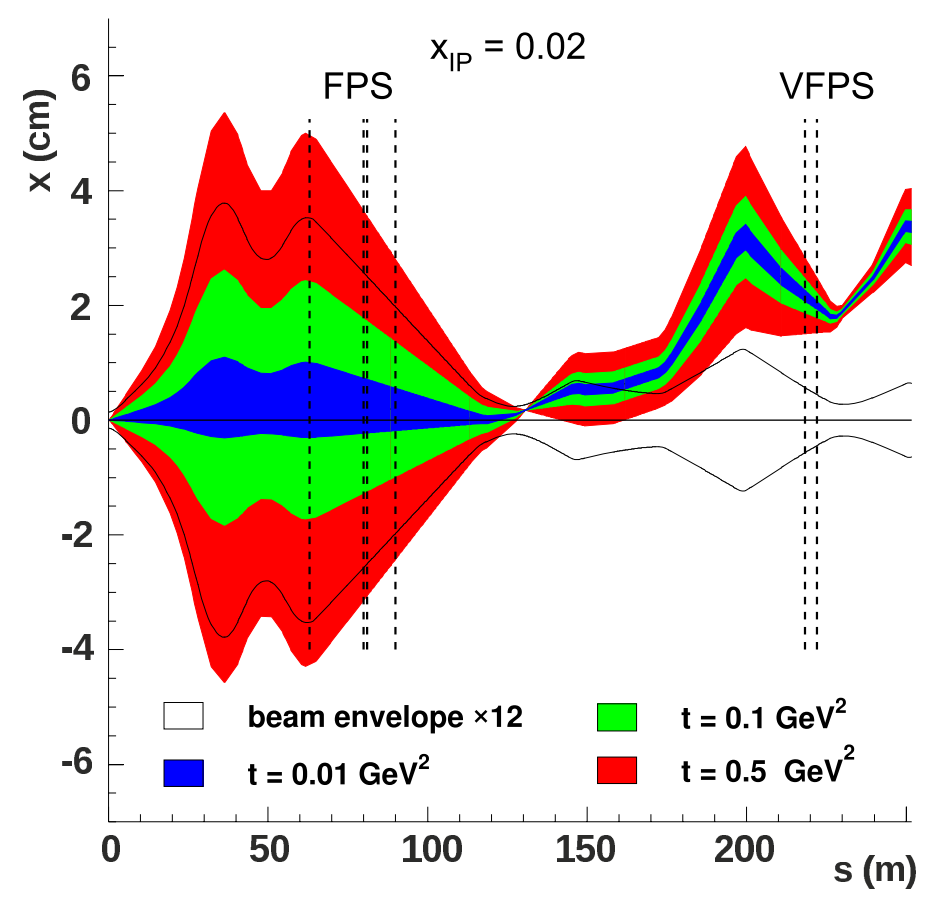

Figure 2. Beam envelope [2] as a function of the distance $s$ to the $\mathrm{H} 1$ vertex in the $x$ projection, for the $p$ beam and diffractive protons at $x_{\mathbb{P}}=0.02$ and $|t|=0.01,0.1$ and $0.5 \mathrm{GeV}^{2}$. The locations of FPS/VFPS stations are indicated by the vertical lines.

The hadronic final state (HFS) is reconstructed using an energy flow algorithm which combines information from the trackers and calorimeters by avoiding double-counting of energies [47, 48].

Jets are reconstructed from the hadronic final state objects using the longitudinally invariant $k_{T}$-jet algorithm [49] with a jet distance parameter $R=1.0$ as implemented in the FastJet package [50]. The massless $p_{T}$-recombination scheme is used. The jet finding algorithm is applied in the $\gamma^{*} p$ frame. In photoproduction this frame is identical to the laboratory frame up to a Lorentz boost along the beam axis.

The jet properties are studied in terms of the transverse energy of the leading jet $E_{T}^{* j \mathrm{j} 1}$ in the $\gamma^{*} p$ frame, of the invariant mass of the dijet system $M_{12}$ and of the pseudorapidity variables $\left|\Delta \eta^{\text {jets }}\right|$ and $\left\langle\eta^{\text {jets }}\right\rangle$ defined in the laboratory frame, where

$$
\begin{aligned}
M_{12}^{2} & =\left(J^{(1)}+J^{(2)}\right)^{2}, \\
\left|\Delta \eta^{\text {jets }}\right| & =\left|\eta^{\text {jet} 1}-\eta^{\text {jet} 2}\right|, \\
\left\langle\eta^{\text {jets }}\right\rangle & =\frac{1}{2}\left(\eta^{\text {jet1 }}+\eta^{\text {jet} 2}\right) .
\end{aligned}
$$

In these definitions, $J^{(1)}$ and $J^{(2)}$ denote the four-momenta of the two leading jets. 


\subsubsection{DIS}

For DIS events the polar angle $\theta_{e}^{\prime}$ and energy $E_{e}^{\prime}$ of the scattered positron are measured in the SpaCal calorimeter. The kinematic reconstruction method introduced in [51] is used

$$
y=y_{D A}+y_{e}^{2}-y_{D A}^{2}, \quad Q^{2}=\frac{4 E_{e}^{2}(1-y)}{\tan ^{2} \frac{\theta_{e}^{\prime}}{2}} .
$$

This method interpolates between $y_{e}$ determined from the scattered positron alone at larger inelasticity and $y_{D A}$ determined using the double angle method at low $y$.

The variable $z_{\mathbb{P}}^{\text {obs }}$ is calculated as

$$
z_{\mathbb{P}}^{\mathrm{obs}}=\frac{Q^{2}+M_{12}^{2}}{Q^{2}+M_{X}^{2}}
$$

\subsubsection{Photoproduction}

In the $\gamma p$ regime the scattered positron leaves the interaction undetected. Therefore the inelasticity $y$ is reconstructed from the hadronic final state

$$
y=\frac{\sum_{i \in X}\left(E_{i}-P_{z, i}\right)}{2 E_{e}},
$$

where $E_{e}$ is the initial positron beam energy.

The observables $x_{\gamma}^{\text {obs }}$ and $z_{\mathbb{P}}^{\text {obs }}$ are calculated from the hadronic final state $X$ as

$$
x_{\gamma}^{\text {obs }}=\frac{\sum_{i \in \text { jets }}\left(E_{i}-P_{z, i}\right)}{\sum_{i \in X}\left(E_{i}-P_{z, i}\right)} \quad \text { and } \quad z_{\mathbb{P}}^{\text {obs }}=\frac{\sum_{i \in \text { jets }}\left(E_{i}+P_{z, i}\right)}{\sum_{i \in X}\left(E_{i}+P_{z, i}\right)},
$$

where the sums in the numerators run over the leading and the sub-leading jet, whereas the sums in the denominators include all objects of the reconstructed hadronic final state.

\subsection{Event selection}

The analysis is based on a data sample corresponding to an integrated luminosity of $30 \mathrm{pb}^{-1}$ for photoproduction and $50 \mathrm{pb}^{-1}$ for DIS collected with the H1 detector in the years 2006 and 2007 with proton and positron beam energies of $920 \mathrm{GeV}$ and $27.6 \mathrm{GeV}$, respectively. The events are triggered on the basis of a coincidence of VFPS signals from both stations, together with conditions on the charged track transverse momenta and track topology in the $\mathrm{H1}$ main detector [52]. The trigger efficiency, calculated using events collected with an independent trigger condition, was found to be about $80 \%$ with negligible dependence on kinematic quantities. This efficiency is well reproduced by the H1 trigger simulation after correcting for an overall normalisation difference of $5 \%$. For the DIS analysis the integrated luminosity is increased using the fact that for most of the DIS events also another trigger based on signals in the SpaCal has fired. Only events with a VFPS track in a fiducial volume of high efficiency are selected [2]. The reconstructed $z$-coordinate of the event vertex is required to be within $30 \mathrm{~cm}$ of the mean $z$-position of the interaction point.

The random overlap of $e p$ events with beam-halo protons detected in the VFPS can constitute a possible background to the VFPS diffractive data sample. In such background 
events the detected proton typically has a small energy loss, not compatible with the energy loss expected from the energy deposited in the main H1 detector. The relative energy loss of the proton detected in VFPS, $x_{\mathbb{P}}^{\mathrm{VFPS}}$, is thus required to be at least $60 \%$ of $x_{\mathbb{P}}^{\mathrm{H} 1}$ measured in the $\mathrm{H} 1$ detector, ${ }^{1} x_{\mathbb{P}}^{\mathrm{VFPS}} / x_{\mathbb{P}}^{\mathrm{H} 1}>0.6$. In addition, $x_{\mathbb{P}}^{\mathrm{H} 1}$ is required to be smaller than 0.04 . The remaining background contamination after applying the above cuts is estimated from data by overlaying events without VFPS activity with VFPS signals recorded independently of any detector activity and is found to be less than $1 \%$ [2].

The scattered positron candidate of an event is identified as the electromagnetic cluster with the highest transverse momentum being well isolated and having a minimum energy of $8 \mathrm{GeV}$. If such a candidate is absent the event is defined as photoproduction.

For the selection of DIS events in this analysis the positron candidate is required to be detected in the SpaCal. The energy $E_{e}^{\prime}$ and polar angle $\theta_{e}^{\prime}$ of the scattered positron are determined from the SpaCal cluster and the interaction vertex reconstructed in the CTD. In order to improve the background rejection, additional requirements on the transverse cluster radius and lower limit to the positron energy are imposed [53]. The quantity $\sum_{i}\left(E_{i}-P_{z, i}\right)$ summed over all HFS particles and the scattered positron, is required to be in the range $35-75 \mathrm{GeV}$. For fully reconstructed neutral current DIS events this quantity is expected to be twice the positron beam energy $(55.2 \mathrm{GeV})$ but is expected to be lower for photoproduction background where the scattered positron escapes undetected. Radiative events where a photon is emitted along the direction of the incident positron beam, also have a reduced $\sum_{i}\left(E_{i}-P_{z, i}\right)$.

The leading and the sub-leading jets are required to have transverse energies $E_{T}^{* \text { jet } 1}>$ $5.5 \mathrm{GeV}$ and $E_{T}^{* j \mathrm{j} 2}>4.0 \mathrm{GeV}$, respectively. These cuts are asymmetric in the transverse energy to restrict the phase space to a region where NLO QCD calculations are reliable [54, 55]. An event is rejected if one of these two jets is outside of $-1<\eta^{\text {jet1,2 }}<2.5$. Events with $z_{\mathbb{P}}^{\text {obs }}$ above 0.8 are excluded to improve the reliability of the comparison between data and theoretical predictions, since the DPDF are determined with a similar $z_{\mathbb{P}}$ restriction.

The DIS events are selected with photon virtualities $4 \mathrm{GeV}^{2}<Q^{2}<80 \mathrm{GeV}^{2}$. Both data samples are restricted to a common $y$ range $0.2<y<0.7$. In table 1 the definitions of both analysis phase spaces are summarised. The photoproduction and DIS data samples contain 3768 and 550 events, respectively. In addition to the event selection summarised in table 1 an event selection is performed extending the phase space in all kinematic variables and other selection requirements to obtain events for an adequate description of migrations at the phase space boundaries.

\subsection{Monte Carlo simulations}

\subsubsection{Correction to the data}

The Monte Carlo (MC) simulation method is used to correct the data for effects of detector acceptance, resolution and detector inefficiencies. All MC samples are passed through a detailed $\mathrm{H} 1$ detector simulation based on the GEANT program [56] and are subjected to the same analysis chain as is used for the data.

\footnotetext{
${ }^{1}$ The variable $x_{\mathbb{P}}^{\mathrm{H} 1}$ is calculated as $x_{\mathbb{P}}^{\mathrm{H} 1}=\frac{Q^{2}+M_{X}^{2}}{y s}$.
} 


\begin{tabular}{|c|c|}
\hline & Photoproduction \\
\hline \multirow{2}{*}{ Event kinematics } & $4 \mathrm{GeV}^{2}<Q^{2}<80 \mathrm{GeV}^{2}$ \\
\hline & $0.2<y<0.7$ \\
\hline \multirow{3}{*}{ Diffractive phase space } & $0.010<x_{\mathbb{P}}<0.024$ \\
\hline & $|t|<0.6 \mathrm{GeV}^{2}$ \\
\hline & $z_{\mathbb{P}}<0.8$ \\
\hline \multirow{3}{*}{ Jet phase space } & $E_{T}^{* j e t 1}>5.5 \mathrm{GeV}$ \\
\hline & $E_{T}^{* \mathrm{jet} 2}>4.0 \mathrm{GeV}$ \\
\hline & $-1<\eta^{\text {jet } 1,2}<2.5$ \\
\hline
\end{tabular}

Table 1. Phase space of the diffractive dijet VFPS measurement for photoproduction and deepinelastic scattering.

Diffractive dijet photoproduction and DDIS events were generated using the RAPGAP MC generator [57]. This generator is based on leading order (LO) parton level QCD matrix elements with a minimum transverse momentum of the outgoing partons of $\hat{p}_{T}^{\min }=1.7 \mathrm{GeV}$. Higher orders are mimicked by initial and final state leading logarithm parton showers. Fragmentation is accounted for using Lund string model [58] as implemented in Pythia MC generator [59]. The H12006 Fit-B DPDF set [4] is used in RAPGAP to describe the density of partons in the diffractively scattered proton. In photoproduction a resolved photon contribution is simulated using the GRV-LO photon distribution function [25]. In addition to a pomeron exchange contribution also a sub-leading reggeon contribution is included, corresponding to about $\sim 2 \%$ of the total cross section. In order to describe the data sufficiently well reweighting functions are applied in $z_{\mathbb{P}}^{\text {obs }}, x_{\mathbb{P}}$ and $t$. The reweighting is different for $\gamma p$ and DIS.

\subsubsection{Correction to theoretical models}

For comparison of QCD calculations with the diffractive measurements, it is necessary to convert the calculated NLO parton level cross sections to the level of stable hadrons by evaluating effects due to hadronisation, fragmentation and the influence of pomeron or photon remnants. The RAPGAP MC generator is used to compute the required hadronisation correction factors for the diffractive dijet calculations. These factors are defined for each measured data point by

$$
1+\delta_{\mathrm{hadr}}^{i}=\frac{\sigma_{i}^{\mathrm{hadr}}}{\sigma_{i}^{\text {part }}}
$$

where the $\sigma_{i}^{\text {hadr }}\left(\sigma_{i}^{\text {part }}\right)$ are the bin-integrated MC cross sections at hadron level (parton level) in a given bin $i$. They reduce the predicted NLO parton level cross sections by typically $\sim 9 \%$ in photoproduction and enhance the cross sections by typically $\sim 2 \%$ in DIS. In photoproduction the hadronisation correction factor is particularly large at the second highest $x_{\gamma}^{\text {obs }}$ bin, where contributions with $x_{\gamma}^{\text {obs }} \sim 1$ at parton level migrate to 
lower values due to hadronisation effects. The hadronisation corrections have uncertainties of $3 \%$ [17]. The hadronisation corrections determined here are applicable to NLO QCD predictions, since a good agreement in shape of the parton level predictions of the MC to the NLO calculations is observed.

In the DIS analysis, the RAPGAP MC generator is also used to correct the measured data for QED radiation effects. The radiative corrections are defined as

$$
1+\delta_{\mathrm{rad}}^{i}=\frac{\sigma_{i}^{\mathrm{nrad}}}{\sigma_{i}^{\mathrm{rad}}}
$$

where $\sigma_{i}^{\text {rad }}\left(\sigma_{i}^{\text {nrad }}\right)$ denote the bin integrated cross sections obtained from RAPGAP when run with (without) simulating QED radiation. The term $\delta_{\text {rad }}^{i}$ is on average compatible with zero with a standard deviation of $4 \%$ within the phase space. Radiative corrections in photoproduction are found to be negligible.

For the comparison with the measurement, the NLO QCD predictions are scaled down by a factor of $0.83[60]$ to account for the contributions from proton dissociation $\left(M_{Y}<\right.$ $1.6 \mathrm{GeV}$ ) absent in the current analysis but included in the extraction of the H12006 Fit-B DPDF set from the inclusive data [4].

\subsection{Cross section measurement}

In order to correct for detector effects, the dijet cross sections are calculated at the level of stable hadrons using a matrix unfolding method [61,62]. The detector response is described by a matrix $\mathbf{A}$ determined from the RAPGAP simulation. It relates the expected vector of event counts, $\left\langle\vec{y}_{\text {rec }}\right\rangle$, to the true event count vector, $\vec{x}_{\text {true }}$, on the level of stable hadrons via the formula $\left\langle\vec{y}_{\text {rec }}\right\rangle=\mathbf{A} \vec{x}_{\text {true }}$.

In order to control migrations at the phase space boundaries also the neighbouring parts of the analysis phase space are taken into account. Of these, the migrations caused by events in which jets have low transverse momenta $E_{T}$, high $x_{\mathbb{P}}$ or low $y$ are most important. Similar unfolding techniques have been applied in other jet-based analyses [10, 11, 63].

An estimator of the true-level event count $\vec{x}_{\text {true }}$ is obtained by minimising a $\chi^{2}$ function (5.12) with respect to $\vec{x}_{\text {true }}$

$\chi^{2}=\chi_{A}^{2}+\tau^{2} \chi_{L}^{2}=\frac{1}{2}\left(\vec{y}_{\text {rec }}-\mathbf{A} \vec{x}_{\text {true }}\right)^{T} \mathbf{V}^{-1}\left(\vec{y}_{\text {rec }}-\mathbf{A} \vec{x}_{\text {true }}\right)+\tau^{2}\left(\vec{x}_{\text {true }}-\vec{x}_{b}\right)^{T} \mathbf{L}^{T} \mathbf{L}\left(\vec{x}_{\text {true }}-\vec{x}_{b}\right)$,

where the matrix $\mathbf{V}$ is the covariance of data. The term $\chi_{A}^{2}$ is a measure of the agreement between $\mathbf{A} \vec{x}_{\text {true }}$ and $\vec{y}_{\text {rec }}$, where $\vec{y}_{\text {rec }}$ is the vector of events counts after subtracting residual background contributions. The regularisation term $\tau^{2} \chi_{L}^{2}$ suppresses large fluctuations of $\vec{x}_{\text {true }}$. The type of the regularisation is defined by the matrix $\mathbf{L}$. In this paper, $\mathbf{L}$ is set to the unity matrix. The vector $\vec{x}_{B}$ defines a bias for the regularisation term, taken from the RAPGAP prediction. The value of the regularisation parameter $\tau$ is chosen using the $L$-curve method as described elsewhere [61].

The bin-integrated cross section for each data point is given by

$$
\sigma_{i}=\frac{x_{\text {true }}^{i}}{\mathcal{L}}\left(1+\delta_{\text {rad }}^{i}\right)
$$


where $\mathcal{L}$ is the integrated luminosity of the data. The radiative corrections $\delta_{\text {rad }}^{i}$ are non-zero only for the DIS case.

\subsection{Systematic uncertainties of the measured cross section}

For each source of systematic uncertainty, a separate response matrix $\mathbf{A}$ is filled and the difference to the nominal matrix $\mathbf{A}$ is propagated through the unfolding procedure. All these individual contributions of systematic uncertainties are then added in quadrature for each bin to obtain the total systematic uncertainty. The following systematic effects are studied:

VFPS calibration The primary source of the VFPS systematic uncertainties is related to an uncertainty of the $x$ and $y$ global track coordinates ${ }^{2}$ with respect to the beam. The actual beam position is measured with help of a beam position monitor $[2,64]$ which has a precision of $160 \mu \mathrm{m}$ in $x$ and $120 \mu \mathrm{m}$ in $y$. The horizontal coordinate $x$ has an additional uncertainty originating from the VFPS calibration procedure, tied to the reconstruction of $x_{\mathbb{P}}$ in the main $\mathrm{H} 1$ detector. The resulting $x$-coordinate uncertainty is $250 \mu \mathrm{m}$.

The time variation of the beam-tilt in $x$ and $y$ introduces an uncertainty of $8 \mu \mathrm{rad}$ for the $x$-tilt and $6 \mu$ rad for the $y$-tilt.

More details on the VFPS reconstruction and its precision are given in [2]. In total, all sources of the VFPS uncertainties affect the integrated cross section by $5.5 \%$ in $\gamma p$ and typically $3.7 \%$ in DIS.

Positron reconstruction In the DIS analysis the uncertainties of the measured positron energy $E_{e}^{\prime}(1 \%)$ and angle $\theta_{e}^{\prime}(1 \mathrm{mrad})$ in the SpaCal calorimeter lead to an uncertainty of the total cross section of $0.4 \%$ and $0.7 \%$, respectively.

Energy scale The uncertainty of the hadronic final state energy calibration is $2 \%$ [44]. It affects the total cross section by $\pm 7.6 \%$ for photoproduction and by $\pm 6.1 \%$ for DIS.

Model uncertainties The influence of the MC model used to unfold the cross sections is studied by varying the kinematic distributions of the RAPGAP MC generator within certain limits while maintaining an acceptable description of the data. For this purpose the shape of the kinematic distributions in $E_{T}^{* j e t 1}, x_{\mathbb{P}}, z_{\mathbb{P}}, x_{\gamma}, y, t$ and $Q^{2}$ are altered by applying multiplicative weights of $\left(E_{T}^{* j e t 1}\right)^{ \pm 0.4}, x_{\mathbb{P}}^{ \pm 0.2}, z_{\mathbb{P}}^{ \pm 0.3}, x_{\gamma}^{ \pm 0.3}$, $y^{ \pm 0.3}, e^{ \pm t}$ and $\left(Q^{2}+0.1 \mathrm{GeV}^{2}\right)^{ \pm 0.2}$, respectively. The largest resulting uncertainties arise from variation of the shape in $t\left(4.5 \%\right.$ in $\gamma p$ and $3.3 \%$ in DIS) and $E_{T}^{* \text { jet } 1}$ (3.5\% in $\gamma p$ and $3.0 \%$ in DIS). The integrated cross section uncertainty due to model dependence is $7 \%$ in $\gamma p$ and $5 \%$ in DIS.

Normalisation uncertainties The following sources of systematic normalisation errors are considered:

- The VFPS track reconstruction efficiency is known to within $2.5 \%$ [2].

\footnotetext{
${ }^{2}$ The global track coordinates are reconstructed by linking the local tracks of the two VFPS stations.
} 

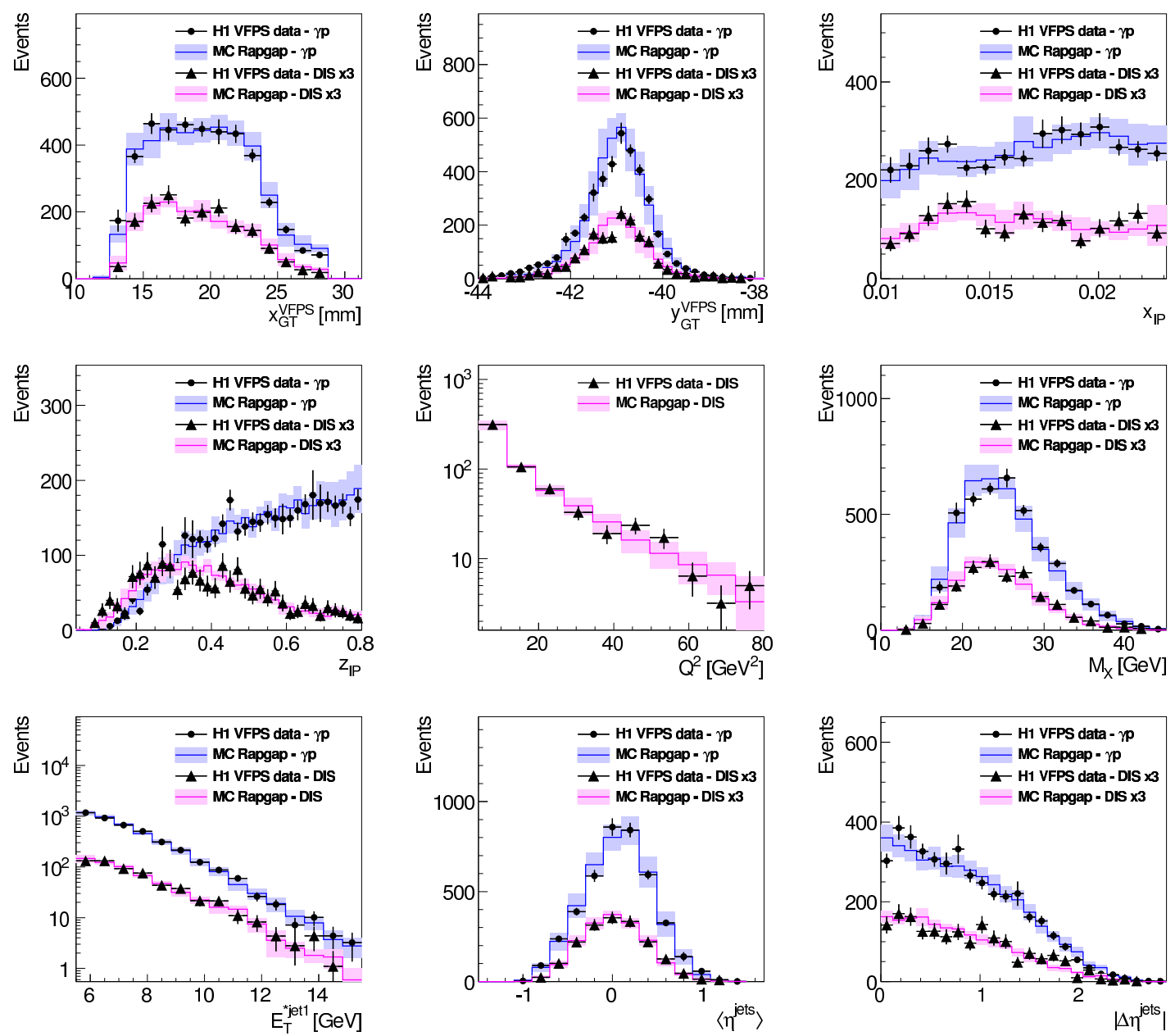

Figure 3. Comparison of the photoproduction data (dots) and DIS data (triangles) with the reweighted RAPGAP MC simulation (solid line) as a function of coordinates $x$ and $y$ in VFPS and the reconstructed variables $x_{\mathbb{P}}, z_{\mathbb{P}}, Q^{2}, E_{T}^{* \text { jet } 1},\left\langle\eta^{\text {jets }}\right\rangle,\left|\Delta \eta^{\text {jets }}\right|$ and $M_{X}$. The systematic uncertainties are shown as bands on the histograms. For better visibility the DIS distributions are scaled up a factor 3 , with the exception of $Q^{2}$ and $E_{T}^{* j e t 1}$.

- The VFPS background originating from interactions of beam particles with the residual gas, producing a proton signal in the VFPS in accidental coincidence with a dijet event in the main $\mathrm{H} 1$ detector is less than $1 \%$ and is treated as a normalisation uncertainty [2].

- The integrated luminosity of the VFPS triggered data is known to within $3 \%[45]$.

- The trigger efficiency has an uncertainty of $5 \%$.

The resulting total normalisation uncertainty amounts to $6 \%$.

Figure 3 displays the distributions of the $x$ - and $y$-coordinates of global tracks in the VFPS, $x_{\mathbb{P}}$ as measured by the VFPS, the variable $z_{\mathbb{P}}, Q^{2}$ for the DIS selection, the mass of 


\begin{tabular}{|c|c|c|}
\hline & PHP & DIS \\
\hline Data $[\mathrm{pb}]$ & $237 \pm 14$ (stat) \pm 31 (syst) & $30.5 \pm 1.6$ (stat) \pm 2.8 (syst) \\
\hline NLO QCD $[\mathrm{pb}]$ & $430_{-98}^{+172}$ (scale) ${ }_{-61}^{+48}(\mathrm{DPDF}) \pm 13$ (hadr) & $28.3_{-6.4}^{+11.4}$ (scale) ${ }_{-4.0}^{+3.0}(\mathrm{DPDF}) \pm 0.8$ (hadr) \\
\hline RAPGAP $[\mathrm{pb}]$ & 180 & 18.0 \\
\hline Data/NLO & $0.551 \pm 0.078$ (data) $)_{-0.149}^{+0.230}$ (theory) & $1.08 \pm 0.11$ (data) ${ }_{-0.29}^{+0.45}$ (theory) \\
\hline
\end{tabular}

Table 2. Integrated $e^{+} p$ diffractive dijet cross sections in $\gamma p$ and DIS compared to NLO QCD calculations using the H12006 Fit-B DPDF set. The measured cross sections are gresented with statistical and systematical uncertainties. For the theoretical predictions the uncertainties from scale variations, from the H12006 Fit-B DPDFs and from the hadronisation corretions are given. The predictions from RAPGAP are also shown. The ratios data/NLO are given in the last row.

the hadronic system $M_{X}$ and the jet variables $E_{T}^{* \text { jet1 }},\left\langle\eta^{\text {jets }}\right\rangle$ and $\left|\Delta \eta^{\text {jets }}\right|$ in comparison to the MC distributions after reweighting and normalising to the data. In all cases the data are well described in shape within systematic errors.

\section{Results}

\subsection{Integrated photoproduction and DIS cross sections}

The integrated $e^{+} p$ diffractive dijet cross sections in the $\gamma p$ and in the DIS regime measured in the kinematic range defined in table 1 are presented together with NLO QCD and RAPGAP predictions in table 2. The total theoretical uncertainty is calculated by using the sign improved quadratic sum of DPDF eigenvectors [11], scale and hadronisation uncertainties. In the DIS regime, the theoretical expectation agrees with the measurement within uncertainties. This confirms the observation made in previous measurements [8-11]. In contrast, the integrated diffractive dijet cross section in photoproduction is overestimated by the NLO QCD theory by almost a factor of two, with considerable theory uncertainty. This observation agrees with the results of previous $\mathrm{H} 1$ analyses in a similar kinematic range $[17,18]$, based on different data sets and using different experimental techniques to select diffractive events. To conclude, the integrated NLO QCD cross section predictions are in disagreement with three independent $\mathrm{H} 1$ measurements of diffractive dijet photoproduction. The MC RAPGAP, based on leading order matrix elements and parton showers, fails to describe the integrated cross sections both in DIS and in photoproduction.

\subsection{Diffractive dijet production in DIS}

The measured differential DIS cross sections as a function of $z_{\mathbb{P}}^{\text {obs }}, x_{\mathbb{P}}, y, Q^{2}$ are given in table 3 and are shown in figure 4 together with the NLO QCD predictions. In table 4 and figure 5 the differential cross sections in DIS are shown as a function of $E_{T}^{* \text { jet } 1},\left\langle\eta^{\text {jets }}\right\rangle$, $\left|\Delta \eta^{\text {jets }}\right|$ and $M_{X}$. The NLO QCD predictions are in good agreement with the measurements within data and theory uncertainties.

The shapes of the NLO predictions are tested using the ratio of data to prediction. A somewhat different shape is observed for data and theory as a function of $Q^{2}$, however the deviations are covered by the uncertainties. Resolved photon [65] and higher twist contributions [66] are expected to change the DIS cross sections at small $Q^{2}$. The predicted 


\begin{tabular}{|c|c|c|c|c|c|c|c|c|}
\hline \multicolumn{2}{|c|}{$\begin{array}{c}\text { integrated } \\
\text { cross section }\end{array}$} & $\begin{array}{c}\sigma \\
{[\mathrm{pb}]}\end{array}$ & $\begin{array}{l}\delta_{\text {stat }} \\
{[\%]} \\
\end{array}$ & $\begin{array}{c}\rho_{\text {stat }} \\
{[\%]}\end{array}$ & $\begin{array}{c}\delta_{\text {syst }} \\
{[\%]}\end{array}$ & $\begin{array}{c}\rho_{\text {syst }} \\
{[\%]}\end{array}$ & $1+\delta_{\mathrm{rad}}$ & $1+\delta_{\text {hadr }}$ \\
\hline & & 30.5 & 5.4 & & 9.0 & & 0.999 & 1.022 \\
\hline$z_{\mathbb{P}}$ & bin & $\begin{array}{c}d \sigma / d z_{\mathbb{P}} \\
{[\mathrm{pb}]}\end{array}$ & $\begin{array}{c}\delta_{\text {stat }} \\
{[\%]}\end{array}$ & $\begin{array}{c}\rho_{\text {stat }} \\
{[\%]}\end{array}$ & $\begin{array}{c}\delta_{\text {syst }} \\
{[\%]}\end{array}$ & $\begin{array}{c}\rho_{\text {syst }} \\
{[\%]}\end{array}$ & $1+\delta_{\mathrm{rad}}$ & $1+\delta_{\text {hadr }}$ \\
\hline $0 \div 0.2$ & 1 & 32.1 & 20.9 & & 16.6 & & 0.916 & 1.084 \\
\hline $0.2 \div 0.4$ & 2 & 59.8 & 12.4 & $\rho_{12}=-17$ & 10.6 & $\rho_{12}=74$ & 1.012 & 1.054 \\
\hline $0.4 \div 0.6$ & 3 & 48.0 & 14.9 & $\rho_{13}=-4 \rho_{23}=-18$ & 9.9 & $\rho_{13}=33 \rho_{23}=79$ & 1.017 & 0.996 \\
\hline $0.6 \div 0.8$ & 4 & 13.9 & 39.0 & $\rho_{14}=4 \rho_{24}=1 \rho_{34}=-37$ & 16.6 & $\rho_{14}=-12 \rho_{24}=28 \rho_{34}=17$ & 1.028 & 0.910 \\
\hline$x_{\mathbb{P}}$ & bin & $\begin{array}{c}d \sigma / d x_{\mathbb{P}} \\
{[\mathrm{pb}]}\end{array}$ & $\begin{array}{c}\delta_{\text {stat }} \\
{[\%]}\end{array}$ & $\begin{array}{c}\rho_{\text {stat }} \\
{[\%]}\end{array}$ & $\begin{array}{l}\delta_{\text {syst }} \\
{[\%]}\end{array}$ & $\begin{array}{c}\rho_{\text {syst }} \\
{[\%]}\end{array}$ & $1+\delta_{\mathrm{rad}}$ & $1+\delta_{\text {hadr }}$ \\
\hline $0.01 \div 0.014$ & 1 & 2250 & 14.3 & & 20.1 & & 0.998 & 1.058 \\
\hline $0.014 \div 0.019$ & 2 & 2210 & 12.8 & $\rho_{12}=-14$ & 14.4 & $\rho_{12}=-33$ & 1.003 & 1.014 \\
\hline $0.019 \div 0.024$ & 3 & 2290 & 12.5 & $\rho_{13}=4 \rho_{23}=-18$ & 12.0 & $\rho_{13}=25 \rho_{23}=41$ & 0.997 & 1.006 \\
\hline$y$ & bin & $\begin{array}{c}d \sigma / d y \\
{[\mathrm{pb}]}\end{array}$ & $\begin{array}{c}\delta_{\text {stat }} \\
{[\%]}\end{array}$ & $\begin{array}{c}\rho_{\text {stat }} \\
{[\%]}\end{array}$ & $\begin{array}{c}\delta_{\text {syst }} \\
{[\%]}\end{array}$ & $\begin{array}{c}\rho_{\text {syst }} \\
{[\%]}\end{array}$ & $1+\delta_{\mathrm{rad}}$ & $1+\delta_{\text {hadr }}$ \\
\hline $0.2 \div 0.32$ & 1 & 76 & 15.7 & & 13.4 & & 1.060 & 0.992 \\
\hline $0.32 \div 0.44$ & 2 & 69.7 & 14.1 & $\rho_{12}=-12$ & 11.7 & $\rho_{12}=86$ & 0.975 & 1.002 \\
\hline $0.44 \div 0.56$ & 3 & 65.4 & 14.7 & $\rho_{13}=0 \rho_{23}=-12$ & 10.7 & $\rho_{13}=63 \rho_{23}=73$ & 0.992 & 1.056 \\
\hline $0.56 \div 0.7$ & 4 & 38.6 & 21.4 & $\rho_{14}=-1 \rho_{24}=1 \rho_{34}=-17$ & 10.4 & $\rho_{14}=39 \rho_{24}=59 \rho_{34}=59$ & 0.948 & 1.084 \\
\hline $\begin{array}{c}Q^{2} \\
{\left[\mathrm{GeV}^{2}\right]}\end{array}$ & bin & $\begin{array}{c}d \sigma / d Q^{2} \\
{\left[\mathrm{pb} / \mathrm{GeV}^{2}\right]}\end{array}$ & $\begin{array}{c}\delta_{\text {stat }} \\
{[\%]}\end{array}$ & $\begin{array}{c}\rho_{\text {stat }} \\
{[\%]}\end{array}$ & $\begin{array}{l}\delta_{\text {syst }} \\
{[\%]}\end{array}$ & $\begin{array}{c}\rho_{\text {syst }} \\
{[\%]}\end{array}$ & $1+\delta_{\mathrm{rad}}$ & $1+\delta_{\text {hadr }}$ \\
\hline $4 \div 5$ & 1 & 4.83 & 23.8 & & 14.7 & & 0.982 & 1.020 \\
\hline $5 \div 7$ & 2 & 2.55 & 21.3 & $\rho_{12}=-17$ & 15.3 & $\rho_{12}=36$ & 1.002 & 1.020 \\
\hline $7 \div 11$ & 3 & 1.66 & 16.1 & $\rho_{13}=3 \rho_{23}=-11$ & 12.1 & $\rho_{13}=80 \rho_{23}=63$ & 0.974 & 1.028 \\
\hline $11 \div 30$ & 4 & 0.520 & 12.1 & $\rho_{14}=1 \rho_{24}=3 \rho_{34}=-4$ & 11.1 & $\rho_{14}=39 \rho_{24}=66 \rho_{34}=71$ & 1.019 & 1.034 \\
\hline $30 \div 80$ & 5 & 0.104 & 19.4 & $\rho_{15}=1 \rho_{25}=2 \rho_{35}=3 \rho_{45}=-1$ & 17.6 & $\rho_{15}=-7 \rho_{25}=23 \rho_{35}=27 \rho_{45}=78$ & 1.036 & 1.013 \\
\hline
\end{tabular}

Table 3. Integrated cross section and bin averaged hadron level differential cross sections as a function of the variables $z_{\mathbb{P}}, x_{\mathbb{P}}, y$ and $Q^{2}$ for diffractive dijet DIS in the phase space detailed in table 1 . For each data point, the statistical $\left(\delta_{\text {stat }}\right)$ and systematic $\left(\delta_{\text {syst }}\right)$ uncertainties and the corresponding correlation coefficients $\left(\rho_{\text {stat }}, \rho_{\text {syst }}\right)$ are given. The hadronisation correction factors $\left(1+\delta_{\text {hadr }}\right)$ applied to the NLO calculations and the radiative corrections $\left(1+\delta_{\text {rad }}\right)$ are also listed. The overall normalisation uncertainty of $6 \%$ is not included in the table. 

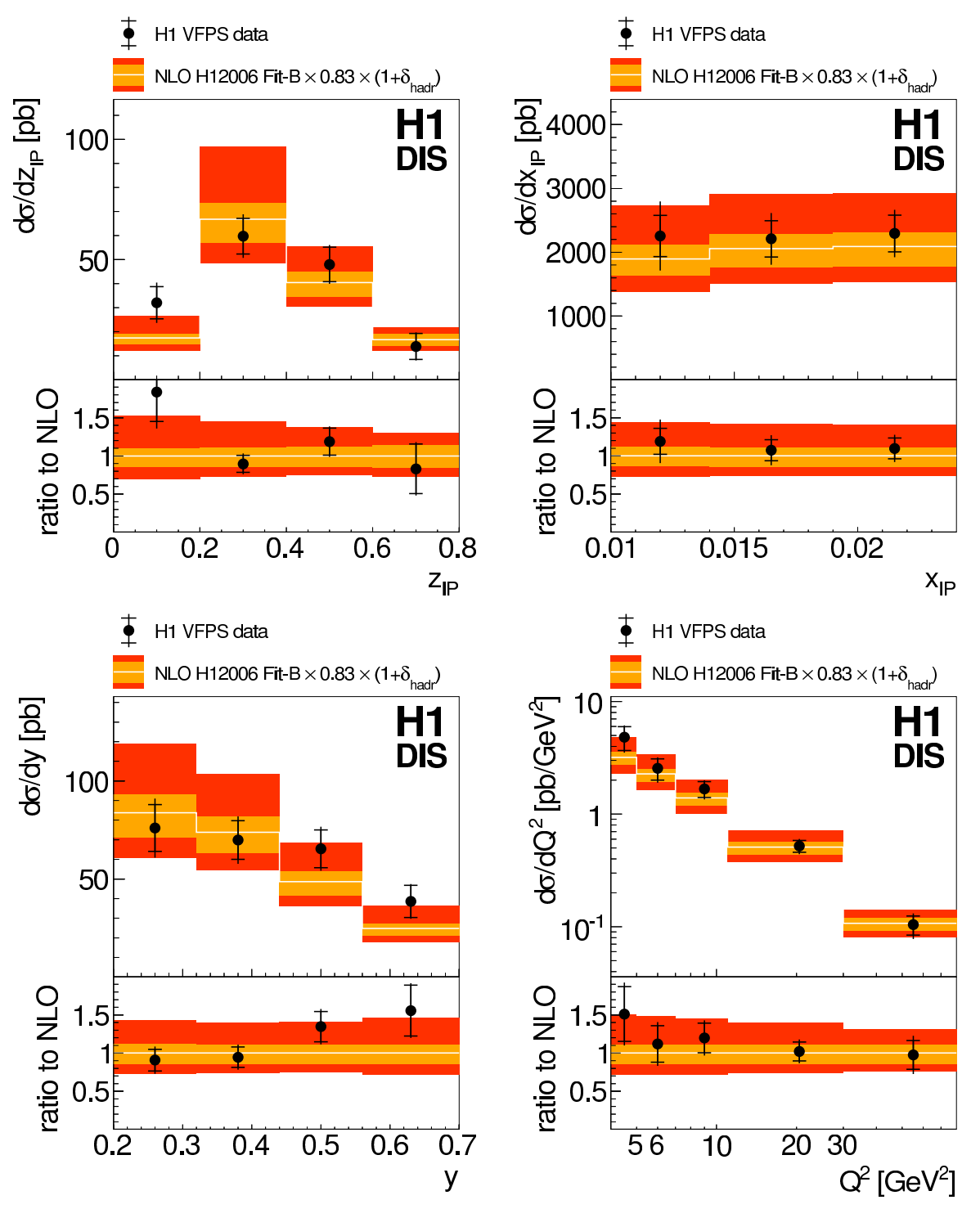

Figure 4. Diffractive dijet DIS cross sections differential in $z_{\mathbb{P}}, x_{\mathbb{P}}, y$ and $Q^{2}$. The inner error bars represent the statistical errors. The outer error bars indicate the statistical and systematic errors added in quadrature. The overall normalisation uncertainty of $6 \%$ is not shown. NLO QCD predictions based on the H12006 Fit-B DPDF set, corrected to the level of stable hadrons, are shown as a white line. They are scaled by a factor 0.83 to account for contributions from protondissociation which are present in the DPDF fit but not in the data. The inner, light shaded band indicates the size of the DPDF uncertainties and hadronisation corrections added in quadrature. The outer, dark shaded band indicates the total NLO uncertainty, also including scale variations by a factor of 0.5 to 2 . For each variable, the cross section is shown in the upper panel, whereas the ratio to the NLO prediction is shown in the lower panel. 


\begin{tabular}{|c|c|c|c|c|c|c|c|c|}
\hline $\begin{array}{l}E_{T}^{* \text { jet1 }} \\
{[\mathrm{GeV}]}\end{array}$ & bin & $\begin{array}{c}d \sigma / d E_{T}^{* \text { jet } 1} \\
{[\mathrm{pb} / \mathrm{GeV}]}\end{array}$ & $\begin{array}{c}\delta_{\text {stat }} \\
{[\%]}\end{array}$ & $\begin{array}{c}\rho_{\text {stat }} \\
{[\%]}\end{array}$ & $\begin{array}{c}\delta_{\text {syst }} \\
{[\%]}\end{array}$ & $\begin{array}{c}\rho_{\text {syst }} \\
{[\%]}\end{array}$ & $1+\delta_{\mathrm{rad}}$ & $1+\delta_{\text {hadr }}$ \\
\hline $5.5 \div 7$ & 1 & 11.24 & 8.3 & & 11.9 & & 0.999 & 1.006 \\
\hline $7 \div 8.5$ & 2 & 5.66 & 16.4 & $\rho_{12}=-4$ & 12.6 & $\rho_{12}=-25$ & 0.986 & 1.034 \\
\hline $8.5 \div 10$ & 3 & 2.55 & 36.0 & $\rho_{13}=-22 \rho_{23}=-21$ & 29.8 & $\rho_{13}=-70 \rho_{23}=80$ & 1.050 & 1.112 \\
\hline $10 \div 14.5$ & 4 & 0.485 & 45.2 & $\rho_{14}=10 \rho_{24}=-14 \rho_{34}=-39$ & 15.0 & $\rho_{14}=-30 \rho_{24}=38 \rho_{34}=42$ & 0.961 & 0.976 \\
\hline $\begin{array}{c}M_{X} \\
{[\mathrm{GeV}]}\end{array}$ & bin & $\begin{array}{l}d \sigma / d M_{X} \\
{[\mathrm{pb} / \mathrm{GeV}]}\end{array}$ & $\begin{array}{c}\delta_{\text {stat }} \\
{[\%]}\end{array}$ & $\begin{array}{c}\rho_{\text {stat }} \\
{[\%]}\end{array}$ & $\begin{array}{c}\delta_{\text {syst }} \\
{[\%]}\end{array}$ & $\begin{array}{c}\rho_{\text {syst }} \\
{[\%]}\end{array}$ & $1+\delta_{\mathrm{rad}}$ & $1+\delta_{\text {hadr }}$ \\
\hline $10 \div 20$ & 1 & 0.20 & 61.5 & & 120.1 & & 1.024 & 0.977 \\
\hline $20 \div 28$ & 2 & 2.06 & 9.5 & $\rho_{12}=-23$ & 10.6 & $\rho_{12}=76$ & 1.026 & 1.021 \\
\hline $28 \div 36$ & 3 & 1.43 & 12.5 & $\rho_{13}=3 \rho_{23}=-18$ & 12.0 & $\rho_{13}=-67 \rho_{23}=-16$ & 0.952 & 1.046 \\
\hline$\left|\Delta \eta^{\text {jets }}\right|$ & bin & $\begin{array}{c}d \sigma / d\left|\Delta \eta^{\text {jets }}\right| \\
{[\mathrm{pb}]}\end{array}$ & $\begin{array}{c}\delta_{\text {stat }} \\
{[\%]}\end{array}$ & $\begin{array}{c}\rho_{\text {stat }} \\
{[\%]}\end{array}$ & $\begin{array}{c}\delta_{\text {syst }} \\
{[\%]}\end{array}$ & $\begin{array}{c}\rho_{\text {syst }} \\
{[\%]}\end{array}$ & $1+\delta_{\mathrm{rad}}$ & $1+\delta_{\text {hadr }}$ \\
\hline $0 \div 0.5$ & 1 & 24.8 & 12.1 & & 10.8 & & 1.015 & 1.004 \\
\hline $0.5 \div 1$ & 2 & 16.0 & 17.6 & $\rho_{12}=-16$ & 12.7 & $\rho_{12}=81$ & 1.002 & 1.046 \\
\hline $1 \div 1.5$ & 3 & 13.1 & 19.9 & $\rho_{13}=4 \rho_{23}=-16$ & 13.2 & $\rho_{13}=96 \rho_{23}=84$ & 0.968 & 1.030 \\
\hline $1.5 \div 2$ & 4 & 7.8 & 28.9 & $\rho_{14}=0 \rho_{24}=5 \rho_{34}=-14$ & 18.4 & $\rho_{14}=38 \rho_{24}=70 \rho_{34}=53$ & 0.966 & 1.030 \\
\hline$\left\langle\eta^{\mathrm{jets}}\right\rangle$ & bin & $\begin{array}{c}d \sigma / d\left\langle\eta^{\text {jets }}\right\rangle \\
{[\mathrm{pb}]}\end{array}$ & $\begin{array}{l}\delta_{\text {stat }} \\
{[\%]} \\
\end{array}$ & $\begin{array}{c}\rho_{\text {stat }} \\
{[\%]}\end{array}$ & $\begin{array}{c}\delta_{\text {syst }} \\
{[\%]} \\
\end{array}$ & $\begin{array}{c}\rho_{\text {syst }} \\
{[\%]}\end{array}$ & $1+\delta_{\mathrm{rad}}$ & $1+\delta_{\text {hadr }}$ \\
\hline$-1 \div-0.45$ & 1 & 10.3 & 21.1 & & 11.1 & & 1.011 & 0.905 \\
\hline$-0.45 \div-0.05$ & 2 & 30.1 & 11.4 & $\rho_{12}=-13$ & 10.6 & $\rho_{12}=42$ & 1.021 & 1.011 \\
\hline$-0.05 \div 0.25$ & 3 & 30.0 & 14.9 & $\rho_{13}=1 \rho_{23}=-14$ & 11.5 & $\rho_{13}=37 \rho_{23}=81$ & 0.994 & 1.056 \\
\hline $0.25 \div 0.65$ & 4 & 9.2 & 32.3 & $\rho_{14}=-2 \rho_{24}=1 \rho_{34}=-16$ & 20.3 & $\rho_{14}=18 \rho_{24}=63 \rho_{34}=71$ & 0.921 & 1.171 \\
\hline
\end{tabular}

Table 4. Bin averaged hadron level differential cross sections for diffractive dijet DIS as a function of the variables $E_{T}^{* \text { jet1 }}, M_{X},\left|\Delta \eta^{\text {jets }}\right|$ and $\left\langle\eta^{\text {jets }}\right\rangle$ in the phase space detailed in table 1 . For each data point, the statistical $\left(\delta_{\text {stat }}\right)$ and systematic $\left(\delta_{\text {syst }}\right)$ uncertainties and the corresponding correlation coefficients $\left(\rho_{\text {stat }}, \rho_{\text {syst }}\right)$ are given. The hadronisation correction factors $\left(1+\delta_{\text {hadr }}\right)$ applied to the NLO calculations and the the radiative corrections $\left(1+\delta_{\mathrm{rad}}\right)$ are also listed. The overall normalisation uncertainty of $6 \%$ is not included in the table. 

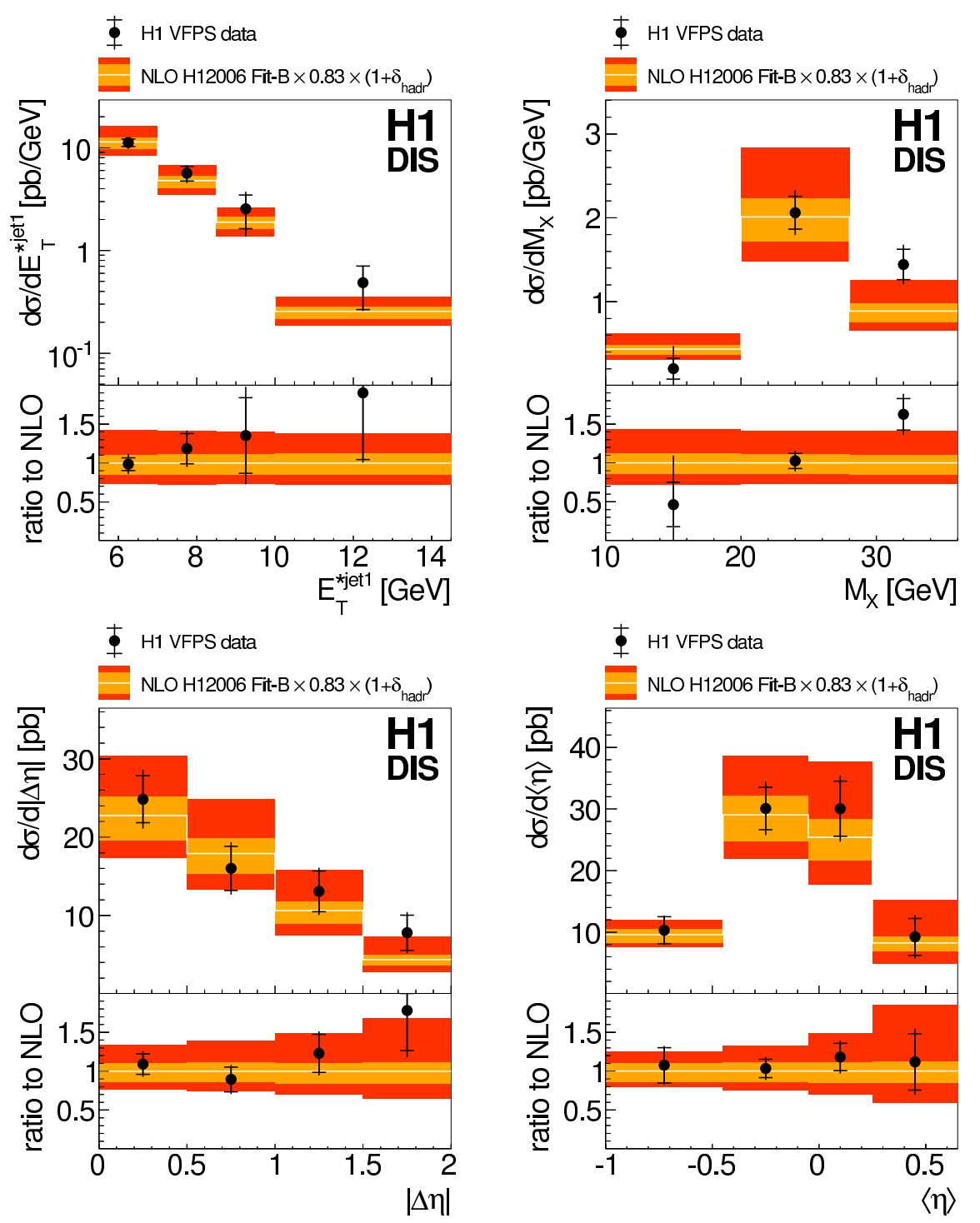

Figure 5. Diffractive dijet DIS cross sections differential in $E_{T}^{* \text { jet } 1}, M_{X},\left|\Delta \eta^{\text {jets }}\right|$ and $\left\langle\eta^{\text {jets }}\right\rangle$. The inner error bars represent the statistical errors. The outer error bars indicate the statistical and systematic errors added in quadrature. Further details are given in the caption of figure 4 .

shape in $y$ also differs from the observation, such that at high $y$ smaller cross sections are predicted than observed. Similar shape deviations in $Q^{2}$ and $y$ have also been observed in a recent measurement of diffractive dijet production based on a large rapidity gap selection [11]. The cross section as a function of $E_{T}^{* j e t 1}$ is observed to be slightly harder than predicted by theory, although still in agreement within uncertainties.

\subsection{Diffractive dijet production in photoproduction}

The measured differential cross sections in the $\gamma p$-regime are given in table 5 and shown in figure 6 as a function of $z_{\mathbb{P}}^{\text {obs }}, x_{\mathbb{P}}, y, x_{\gamma}^{\text {obs }}$ together with the NLO QCD calculations. The differential cross sections for the variables $E_{T}^{* \text { jet } 1},\left\langle\eta^{\text {jets }}\right\rangle,\left|\Delta \eta^{\text {jets }}\right|$ and $M_{X}$ are given 
in table 6 and shown in figure 7. The relative statistical uncertainties in photoproduction are in most cases smaller than in the case of deep-inelastic scattering. The NLO QCD predictions agree well with the measured distributions in shape but overestimate the dijet cross sections in normalisation, as already discussed for the integrated cross sections. In particular there is no significant dependence of the data to theory ratio on the variables $z_{\mathbb{P}}^{\text {obs }}, x_{\gamma}^{\text {obs }}$ and $E_{T}^{* j e t 1}$ which are sensitive to the DPDF and to the presence of a diffractive exchange remnant. These results are in qualitative agreement with previous H1 measurements $[17,18]$. Using the AFG [26] photon PDF as an alternative the predicted integrated cross section is reduced by $6 \%$. As visible in figures 6 and 7 , the shapes of the distributions depend only little on the choice of the photon PDF.

\subsection{Comparison of dijet cross sections in diffractive photoproduction and DIS}

The conclusions made in previous sections about the normalisation problems of the NLO calculations in diffractive photoproduction suffer from large theoretical uncertainties. This situation is summarised in figure 8 , where the ratio of observed cross section to expectation is shown as a function of $Q^{2}$, also including an extra bin for the cross section in the photoproduction regime, $0<Q^{2}<2 \mathrm{GeV}^{2}$. No significant deviation from unity is observed for the suppression factor ${ }^{3}$ as a function of $Q^{2}$ in the DIS regime, whereas the NLO calculation fails to describe the measurement in the photoproduction region. For comparison, also the ratio of the RAPGAP prediction to the NLO calculation is shown. RAPGAP is off in normalisation and predicts a shape in $Q^{2}$ which differs from the NLO calculation.

In a refined method for studying deviations of the NLO QCD predictions from photoproduction data the cross sections measured in the $\gamma p$-regime are divided by the corresponding cross sections in DIS. In such ratios most of the data systematic uncertainties are reduced, with the exception of the model uncertainties which are uncorrelated between $\gamma p$ and DIS. Similarly, theoretical uncertainties cancel to a large extent. This is true for the DPDF uncertainties as well as for scale variations, if the NLO QCD scales are varied simultaneously for photoproduction and DIS. The hadronisation corrections, however, are taken to be uncorrelated between DIS and photoproduction, such that they amount to about $\sqrt{2} \times 3 \%$ in the ratio of the integrated cross section. The resulting cross section ratios of photoproduction to DIS are summarised in table 7. The double-ratio of photoproduction to DIS, data to NLO, is also given and shown in figure 9. Due to the reduced theoretical uncertainty the double ratio deviates significantly from unity indicating that factorisation does not hold in diffractive dijet photoproduction with respect to the same process in DIS. This statement is valid within the theoretical framework applied in this paper and under the assumption that the scale must be varied simultaneously for the DIS and $\gamma \mathrm{p}$ calculations, which leads to cancellations of the respective uncertainties in the ratio. Higher order corrections may change this picture. As an estimate of the possible size of such corrections the difference between leading-order and NLO calculations scaled by $\alpha_{s} / 2$ may be taken, which amounts to $5 \%$. When changing the photon PDF from the GRV PDF set to the AFG PDF set a rise in the double ratio of $6 \%$ is observed. Using

\footnotetext{
${ }^{3}$ The suppression factor is defined as a ratio of data and NLO QCD cross section.
} 

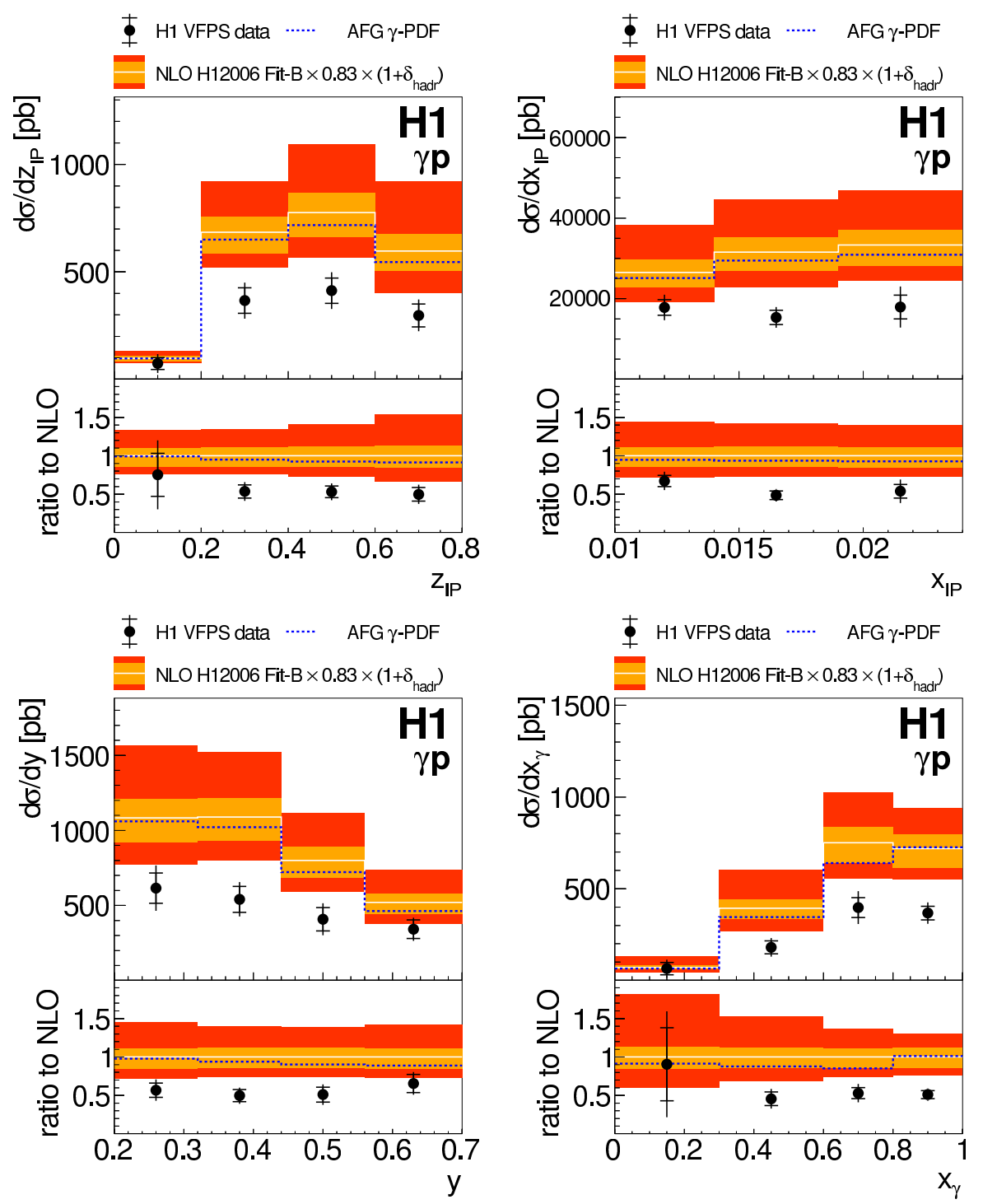

Figure 6. Diffractive dijet $e p$ cross sections in the photoproduction kinematic range differential in $z_{\mathbb{P}}, x_{\mathbb{P}}, y$ and $x_{\gamma}$. The inner error bars represent the statistical errors. The outer error bars indicate the statistical and systematic errors added in quadrature. The overall normalisation uncertainty of $6 \%$ is not shown. NLO QCD predictions based on the H12006 Fit-B DPDF set and the GRV $\gamma$-PDF set, corrected to the level of stable hadrons, are shown as a white line. They are scaled by a factor 0.83 to account for contributions from proton-dissociation which are present in the DPDF fit but not in the data. The inner, light shaded band indicates the size of the DPDF uncertainties and hadronisation corrections added in quadrature. The outer, dark shaded band indicates the total NLO uncertainty, also including scale variations by a factor of 0.5 to 2 . A variant of the NLO calculation using the AFG $\gamma$-PDF set is shown as a dashed line. For each variable, the cross section is shown in the upper panel, whereas the ratio to the NLO prediction is shown in the lower panel. 


\begin{tabular}{|c|c|c|c|c|c|c|c|}
\hline \multicolumn{2}{|c|}{$\begin{array}{c}\text { integrated } \\
\text { cross section }\end{array}$} & $\begin{array}{c}\sigma \\
{[\mathrm{pb}]}\end{array}$ & $\begin{array}{l}\delta_{\text {stat }} \\
{[\%]}\end{array}$ & $\rho_{\text {stat }}$ & $\begin{array}{l}\delta_{\text {syst }} \\
{[\%]}\end{array}$ & $\rho_{\text {syst }}$ & $1+\delta_{\text {hadr }}$ \\
\hline & & 237 & 5.7 & & 13.0 & & 0.906 \\
\hline$z_{\mathbb{P}}$ & bin & $\begin{array}{c}d \sigma / d z_{\mathbb{P}} \\
{[\mathrm{pb}]}\end{array}$ & $\begin{array}{l}\delta_{\text {stat }} \\
{[\%]}\end{array}$ & $\rho_{\text {stat }}$ & $\begin{array}{c}\delta_{\text {syst }} \\
{[\%]}\end{array}$ & $\rho_{\text {syst }}$ & $1+\delta_{\text {hadr }}$ \\
\hline $0 \div 0.2$ & 1 & 73 & 37.2 & & 47.0 & & 0.754 \\
\hline $0.2 \div 0.4$ & 2 & 366 & 16.1 & $\rho_{12}=-19$ & 17.3 & $\rho_{12}=65$ & 0.833 \\
\hline $0.4 \div 0.6$ & 3 & 413 & 14.3 & $\rho_{13}=18 \rho_{23}=-33$ & 16.1 & $\rho_{13}=32 \rho_{23}=83$ & 0.928 \\
\hline $0.6 \div 0.8$ & 4 & 298 & 17.9 & $\rho_{14}=-3 \rho_{24}=22 \rho_{34}=-24$ & 18.3 & $\rho_{14}=-16 \rho_{24}=41 \rho_{34}=81$ & 1.017 \\
\hline$x_{\mathbb{P}}$ & bin & $\begin{array}{c}d \sigma / d x_{\mathbb{P}} \\
{[\mathrm{pb}]}\end{array}$ & $\begin{array}{l}\delta_{\text {stat }} \\
{[\%]}\end{array}$ & $\begin{array}{c}\rho_{\text {stat }} \\
{[\%]}\end{array}$ & $\begin{array}{c}\delta_{\text {syst }} \\
{[\%]}\end{array}$ & $\begin{array}{c}\rho_{\text {syst }} \\
{[\%]}\end{array}$ & $1+\delta_{\text {hadr }}$ \\
\hline $0.01 \div 0.014$ & 1 & 17800 & 10.8 & & 15.4 & & 0.933 \\
\hline $0.014 \div 0.019$ & 2 & 15300 & 11.5 & $\rho_{12}=2$ & 13.7 & $\rho_{12}=81$ & 0.916 \\
\hline $0.019 \div 0.024$ & 3 & 17900 & 16.3 & $\rho_{13}=13 \rho_{23}=-17$ & 24.0 & $\rho_{13}=35 \rho_{23}=29$ & 0.882 \\
\hline$y$ & bin & $\begin{array}{c}d \sigma / d y \\
{[\mathrm{pb}]}\end{array}$ & $\begin{array}{l}\delta_{\text {stat }} \\
{[\%]}\end{array}$ & $\begin{array}{c}\rho_{\text {stat }} \\
{[\%]}\end{array}$ & $\begin{array}{c}\delta_{\text {syst }} \\
{[\%]}\end{array}$ & $\begin{array}{c}\rho_{\text {syst }} \\
{[\%]}\end{array}$ & $1+\delta_{\text {hadr }}$ \\
\hline $0.2 \div 0.32$ & 1 & 620 & 16.4 & & 19.1 & & 0.858 \\
\hline $0.32 \div 0.44$ & 2 & 541 & 15.9 & $\rho_{12}=-12$ & 15.2 & $\rho_{12}=70$ & 0.914 \\
\hline $0.44 \div 0.56$ & 3 & 408 & 19.1 & $\rho_{13}=21 \rho_{23}=-42$ & 18.6 & $\rho_{13}=37 \rho_{23}=70$ & 0.957 \\
\hline $0.56 \div 0.7$ & 4 & 342 & 18.0 & $\rho_{14}=3 \rho_{24}=33 \rho_{34}=-49$ & 14.7 & $\rho_{14}=53 \rho_{24}=80 \rho_{34}=64$ & 0.913 \\
\hline$x_{\gamma}$ & bin & $\begin{array}{c}d \sigma / d x_{\gamma} \\
{[\mathrm{pb}]}\end{array}$ & $\begin{array}{l}\delta_{\text {stat }} \\
{[\%]}\end{array}$ & $\begin{array}{c}\rho_{\text {stat }} \\
{[\%]}\end{array}$ & $\begin{array}{c}\delta_{\text {syst }} \\
{[\%]}\end{array}$ & $\begin{array}{c}\rho_{\text {syst }} \\
{[\%]}\end{array}$ & $1+\delta_{\text {hadr }}$ \\
\hline $0 \div 0.3$ & 1 & 65 & 52.5 & & 55.5 & & 0.654 \\
\hline $0.3 \div 0.6$ & 2 & 180 & 19.6 & $\rho_{12}=5$ & 21.1 & $\rho_{12}=20$ & 0.884 \\
\hline $0.6 \div 0.8$ & 3 & 397 & 13.7 & $\rho_{13}=13 \rho_{23}=-2$ & 18.8 & $\rho_{13}=-5 \rho_{23}=78$ & 1.536 \\
\hline $0.8 \div 1$ & 4 & 367 & 10.1 & $\rho_{14}=2 \rho_{24}=16 \rho_{34}=-21$ & 13.5 & $\rho_{14}=-29 \rho_{24}=64 \rho_{34}=80$ & 0.683 \\
\hline
\end{tabular}

Table 5. Integrated diffractive dijet $e p$ cross section and bin averaged hadron level differential diffractive dijet $e p$ cross sections as a function of the variables $z_{\mathbb{P}}, x_{\mathbb{P}}, y$ and $x_{\gamma}$ for the dijet photoproduction kinematic range in the phase space detailed in table 1 . For each data point, the statistical $\left(\delta_{\text {stat }}\right)$ and systematic $\left(\delta_{\text {syst }}\right)$ uncertainties, the corresponding correlation coefficients $\left(\rho_{\text {stat }}, \rho_{\text {syst }}\right)$ and the hadronisation correction factors $\left(1+\delta_{\text {hadr }}\right)$ applied to the NLO calculations are given. The overall normalisation uncertainty of $6 \%$ is not included in the table. 


\begin{tabular}{|c|c|c|c|c|c|c|c|}
\hline $\begin{array}{l}E_{T}^{* \text { jet1 }} \\
{[\mathrm{GeV}]}\end{array}$ & bin & $\begin{array}{l}d \sigma / d E_{T}^{* \text { jet1 }} \\
{[\mathrm{pb} / \mathrm{GeV}]}\end{array}$ & \begin{tabular}{|c|}
$\delta_{\text {stat }}$ \\
{$[\%]$}
\end{tabular} & $\begin{array}{c}\rho_{\text {stat }} \\
{[\%]}\end{array}$ & $\begin{array}{c}\delta_{\text {syst }} \\
{[\%]}\end{array}$ & $\begin{array}{c}\rho_{\text {syst }} \\
{[\%]}\end{array}$ & $1+\delta_{\text {hadr }}$ \\
\hline $5.5 \div 7$ & 1 & 91 & 14.9 & & 15.1 & & 0.877 \\
\hline $7 \div 8.5$ & 2 & 45.6 & 21.1 & $\rho_{12}=-54$ & 17.3 & $\rho_{12}=42$ & 0.991 \\
\hline $8.5 \div 10$ & 3 & 11.2 & 50.0 & $\rho_{13}=28 \rho_{23}=-69$ & 25.3 & $\rho_{13}=76 \rho_{23}=25$ & 0.956 \\
\hline $10 \div 14.5$ & 4 & 1.15 & 63.8 & $\rho_{14}=-9 \rho_{24}=32 \rho_{34}=-63$ & 66.4 & $\rho_{14}=44 \rho_{24}=90 \rho_{34}=28$ & 0.840 \\
\hline $\begin{array}{c}M_{X} \\
{[\mathrm{GeV}]}\end{array}$ & bin & $\begin{array}{l}d \sigma / d M_{X} \\
{[\mathrm{pb} / \mathrm{GeV}]}\end{array}$ & $\begin{array}{c}\delta_{\text {stat }} \\
{[\%]}\end{array}$ & $\begin{array}{l}\rho_{\text {stat }} \\
{[\%]}\end{array}$ & $\begin{array}{c}\delta_{\text {syst }} \\
{[\%]}\end{array}$ & $\begin{array}{l}\rho_{\text {syst }} \\
{[\%]}\end{array}$ & $1+\delta_{\text {hadr }}$ \\
\hline $10 \div 20$ & 1 & 2.47 & 32.3 & & 30.5 & & 0.899 \\
\hline $20 \div 28$ & 2 & 13.2 & 12.7 & $\rho_{12}=-27$ & 14.7 & $\rho_{12}=9$ & 0.925 \\
\hline $28 \div 36$ & 3 & 12.4 & 13.0 & $\rho_{13}=16 \rho_{23}=-28$ & 18.0 & $\rho_{13}=-43 \rho_{23}=59$ & 0.933 \\
\hline$\left|\Delta \eta^{\text {jets }}\right|$ & bin & $\begin{array}{c}d \sigma / d\left|\Delta \eta^{\text {jets }}\right| \\
{[\mathrm{pb}]}\end{array}$ & $\begin{array}{c}\delta_{\text {stat }} \\
{[\%]}\end{array}$ & $\begin{array}{c}\rho_{\text {stat }} \\
{[\%]}\end{array}$ & $\begin{array}{c}\delta_{\text {syst }} \\
{[\%]}\end{array}$ & $\begin{array}{c}\rho_{\text {syst }} \\
{[\%]}\end{array}$ & $1+\delta_{\text {hadr }}$ \\
\hline $0 \div 0.5$ & 1 & 171 & 8.8 & & 12.1 & & 0.872 \\
\hline $0.5 \div 1$ & 2 & 147 & 11.9 & $\rho_{12}=3$ & 14.1 & $\rho_{12}=96$ & 0.905 \\
\hline $1 \div 1.5$ & 3 & 93 & 14.5 & $\rho_{13}=17 \rho_{23}=-5$ & 18.4 & $\rho_{13}=85 \rho_{23}=94$ & 0.936 \\
\hline $1.5 \div 2$ & 4 & 41 & 25.5 & $\rho_{14}=13 \rho_{24}=18 \rho_{34}=2$ & 29.4 & $\rho_{14}=79 \rho_{24}=89 \rho_{34}=95$ & 0.978 \\
\hline$\left\langle\eta^{\text {jets }}\right\rangle$ & bin & $\begin{array}{c}d \sigma / d\left\langle\eta^{\text {jets }}\right\rangle \\
{[\mathrm{pb}]}\end{array}$ & \begin{tabular}{|c|}
$\delta_{\text {stat }}$ \\
{$[\%]$} \\
\end{tabular} & $\begin{array}{c}\rho_{\text {stat }} \\
{[\%]}\end{array}$ & $\begin{array}{c}\delta_{\text {syst }} \\
{[\%]} \\
\end{array}$ & $\begin{array}{c}\rho_{\text {syst }} \\
{[\%]} \\
\end{array}$ & $1+\delta_{\text {hadr }}$ \\
\hline$-1 \div-0.45$ & 1 & 48.4 & 13.3 & & 13.0 & & 0.795 \\
\hline$-0.45 \div-0.05$ & 2 & 175 & 10.1 & $\rho_{12}=1$ & 12.8 & $\rho_{12}=81$ & 0.881 \\
\hline$-0.05 \div 0.25$ & 3 & 264 & 9.3 & $\rho_{13}=2 \rho_{23}=0$ & 13.6 & $\rho_{13}=71 \rho_{23}=88$ & 0.971 \\
\hline $0.25 \div 0.65$ & 4 & 134 & 16.6 & $\rho_{14}=3 \rho_{24}=3 \rho_{34}=-6$ & 22.3 & $\rho_{14}=46 \rho_{24}=79 \rho_{34}=90$ & 0.976 \\
\hline
\end{tabular}

Table 6. Bin averaged hadron level differential diffractive dijet ep cross sections in the photoproduction kinematic range as a function of the variables $E_{T}^{* \text { jet } 1}, M_{X},\left|\Delta \eta^{\text {jets }}\right|$ and $\left\langle\eta^{\text {jets }}\right\rangle$ in the phase space detailed in table 1 . For each data point, the statistical $\left(\delta_{\text {stat }}\right)$ and systematic $\left(\delta_{\text {syst }}\right)$ uncertainties, the corresponding coefficients $\left(\rho_{\text {stat }}, \rho_{\text {syst }}\right)$ and the hadronisation correction factors $\left(1+\delta_{\text {hadr }}\right)$ applied to the NLO calculations are given. The overall normalisation uncertainty of $6 \%$ is not included in the table. 

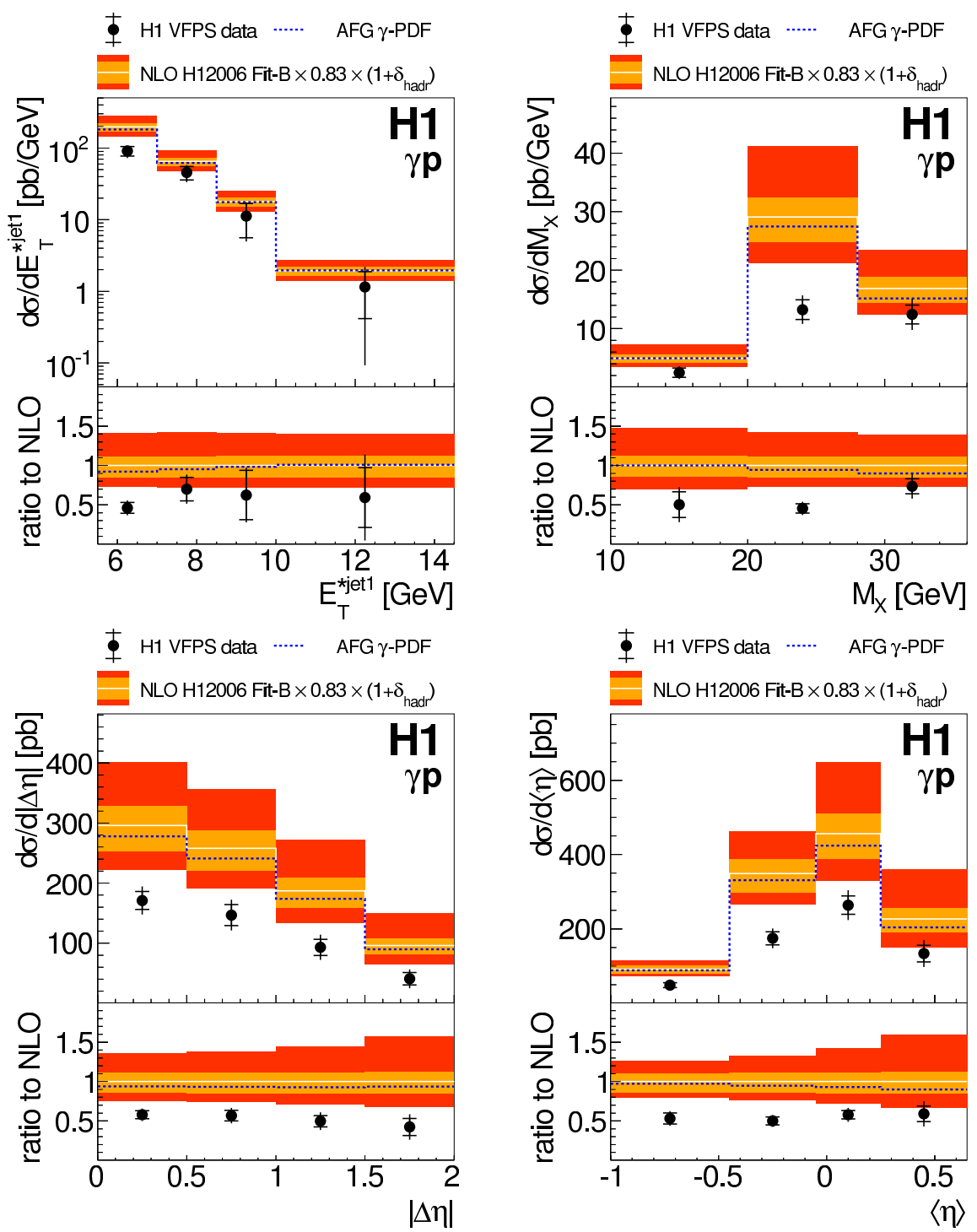

Figure 7. Diffractive dijet $e p$ cross sections in the photoproduction kinematic range differential in $E_{T}^{* \text { jet } 1}, M_{X},\left|\Delta \eta^{\text {jets }}\right|$ and $\left\langle\eta^{\text {jets }}\right\rangle$. The inner error bars represent the statistical errors. The outer error bars indicate the statistical and systematic errors added in quadrature. Further details are given in the caption of figure 6 .

$\mu^{2}=E_{T}^{* j e t 1^{2}}+Q^{2} / 4$ as the scale choice leads to an increase of the double ratio by $7 \%$. The observed suppression agrees with previous $\mathrm{H} 1$ results $[17,18]$. It is worth mentioning that the suppression is now measured at HERA both in processes with an identified leading proton and in processes with a large rapidity gap selection, so possible contributions from proton-dissociative processes alone are excluded as an explanation.

Possible shape dependencies of the suppression are studied using cross section ratios of photoproduction to DIS differential in the variables $\left|\Delta \eta^{\text {jets }}\right|, y, z_{\mathbb{P}}$ and $E_{T}^{* j e t 1}$, as given in table 8. The data ratios as a function of $\left|\Delta \eta^{\text {jets }}\right|, y, z_{\mathbb{P}}$ and $E_{T}^{* \text { jet1 }}$ are shown in figure 10 together with predictions from NLO QCD and RAPGAP. Within uncertainties the corre- 


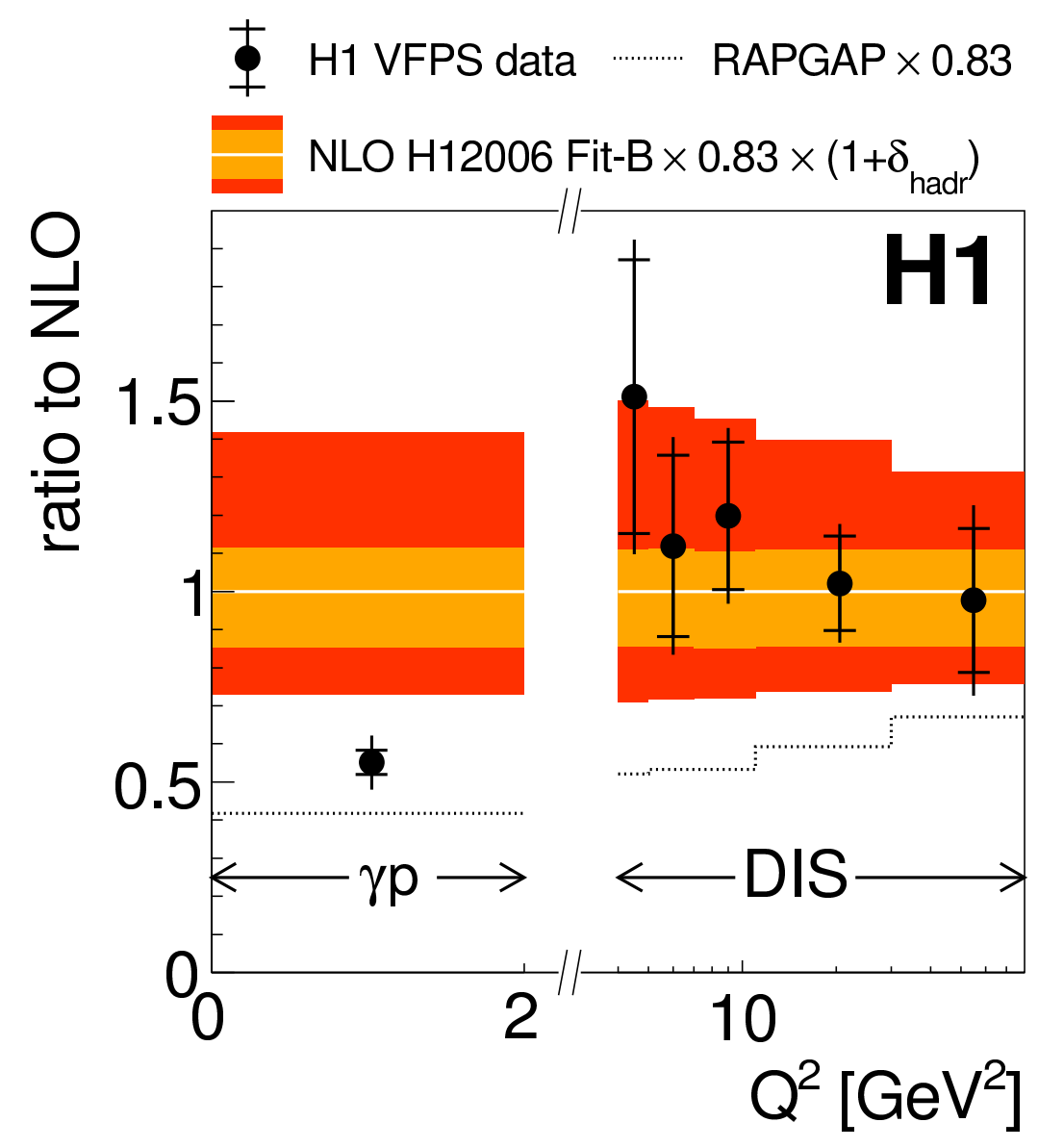

Figure 8. Diffractive dijet cross sections in the $\gamma p$ - and in the DIS regime normalised to the NLO calculation as a function of the photon virtuality $Q^{2}$. The inner error bars represent the statistical errors. The outer error bars indicate the statistical and systematic errors added in quadrature. The data points are displayed at the geometrical bin centre. The NLO QCD predictions are based on the H12006 Fit-B DPDF set and, in case of photoproduction, on the GRV $\gamma$-PDF set, corrected to the level of stable hadrons. They are scaled by a factor 0.83 to account for contributions from protondissociation which are present in the DPDF fit but not in the data. The inner, light shaded band indicates the size of the DPDF uncertainties and hadronisation corrections added in quadrature. The outer, dark shaded band indicates the total NLO uncertainty, also including scale variations by a factor of 0.5 to 2 . Also shown is the ratio of the RAPGAP MC to the NLO prediction.

sponding double ratios, data to NLO QCD, are constant throughout the measured $\left|\Delta \eta^{\text {jets }}\right|$, $y, z_{\mathbb{P}}$ and $E_{T}^{* \text { jet} 1}$ ranges. The largest deviations from a constant are observed for $\left|\Delta \eta^{\text {jets }}\right|$, but the limited experimental precision does not allow for strong conclusions to be made. A linear fit of the double-ratio as a function of $\left|\Delta \eta^{\text {jets }}\right|$ with a constant, taking into account all experimental uncertainties and their correlations, has a least-squares sum of $\chi^{2}=5.3$ at 3 degrees of freedom. This corresponds to a fit probablity of $15 \%$. 


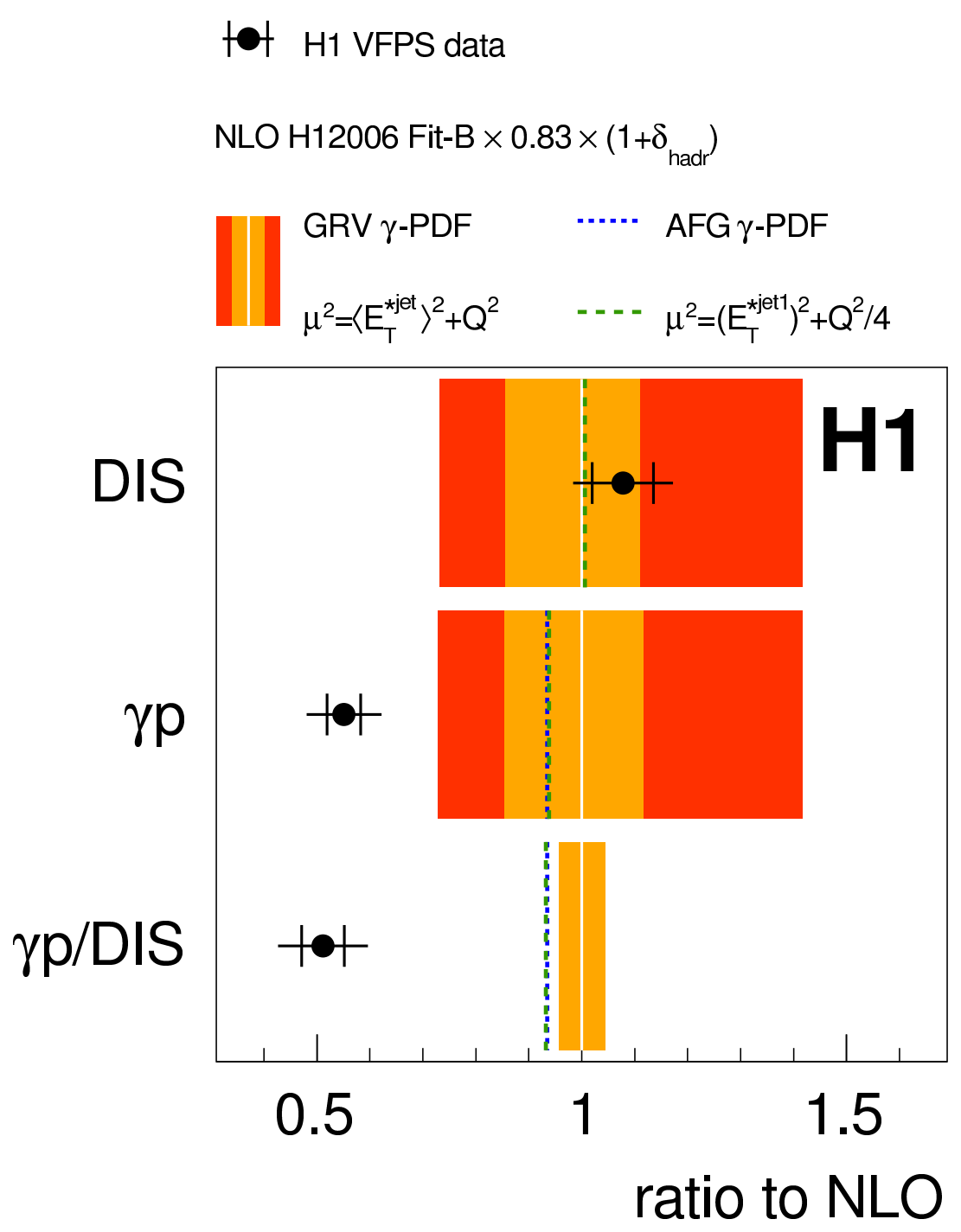

Figure 9. Diffractive dijet DIS and photoproduction cross sections normalised to the NLO calculation. Also shown is the double ratio of photoproduction to DIS cross sections, normalised to the corresponding ratio of NLO predictions. The inner error bars represent the statistical errors. The outer error bars indicate the statistical and systematic errors added in quadrature. The NLO QCD predictions are based on the H12006 Fit-B DPDF set and GRV $\gamma$-PDF, corrected to the level of stable hadrons. They are scaled by a factor 0.83 to account for contributions from proton-dissociation which are present in the DPDF fit but not in the data. The inner, light shaded band indicates the size of the DPDF uncertainties and hadronisation corrections added in quadrature. The outer, dark shaded band indicates the total NLO uncertainty, also including scale variations by a factor of 0.5 to 2. Variants of the NLO calculation, normalised to the default NLO prediction, are also shown: the effect of using the AFG $\gamma$-PDF parametrisation is studied in photoproduction. An alternative functional form of the scale is studied both in DIS and in photoproduction. 

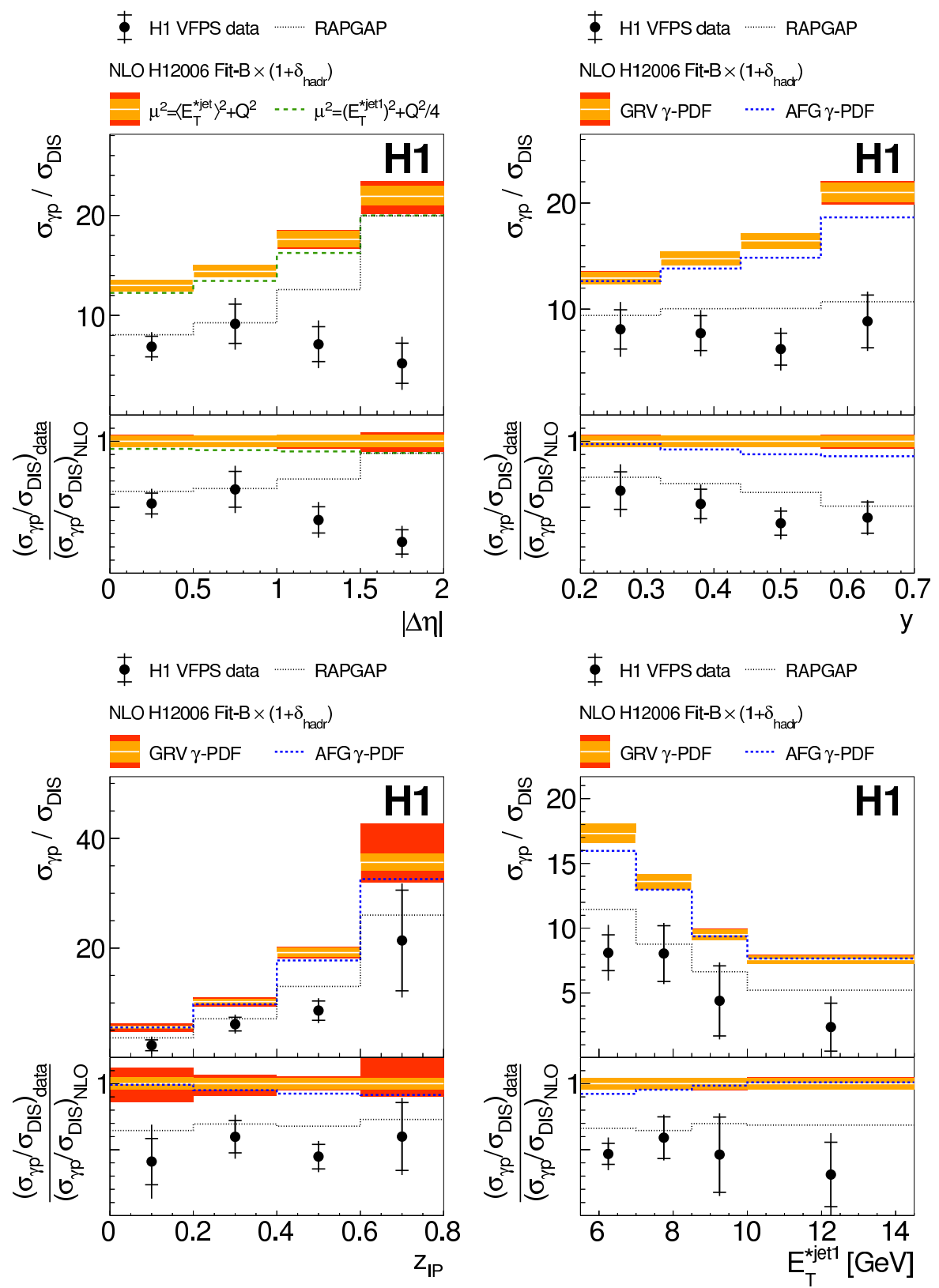

Figure 10. Ratios of diffractive dijet photoproduction to DIS cross sections differential in $|\Delta \eta|, y$, $z_{\mathbb{P}}$ and $E_{T}^{* j e t 1}$. The inner error bars represent the statistical errors. The outer error bars indicate the statistical and systematic errors added in quadrature. The NLO QCD predictions are based on the H12006 Fit-B DPDF set and GRV $\gamma$-PDF, corrected to the level of stable hadrons. They are scaled by a factor 0.83 to account for contributions from proton-dissociation which are present in the DPDF fit but not in the data. The inner, light shaded band indicates the size of the DPDF uncertainties and hadronisation corrections added in quadrature. The outer, dark shaded band indicates the total NLO uncertainty, also including scale variations by a factor of 0.5 to 2 . Variants of the NLO calculation are also shown. An alternative functional form of the scale is studied differential in $|\Delta \eta|$. The effect of using the AFG $\gamma$-PDF parametrisation is studied differential in $y, z_{\mathbb{P}}$ and $E_{T}^{* j e t 1}$. 


\begin{tabular}{|l|l|}
\hline & \multicolumn{2}{|c|}{ Ratio of photoproduction to DIS } \\
\hline Data & $7.78 \pm 0.60$ (stat) \pm 1.14 (syst) \\
NLO QCD & $15.21_{-0.04}^{+0.00}$ (scale) $\quad{ }_{-0.10}^{+0.21}$ (DPDF) \pm 0.65 (hadr) \\
& $14.22 \quad$ with AFG $\gamma \mathrm{PDF}$ \\
& $14.17 \quad$ with scale $\mu^{2}=\left(E_{T}^{* \text { jet1 }}\right)^{2}+Q^{2} / 4$ \\
\hline Data/NLO & $0.511 \pm 0.085$ (data) ${ }_{-0.021}^{+0.022}$ (theory) \\
& $0.547 \quad$ with AFG $\gamma \mathrm{PDF}$ \\
& $0.548 \quad$ with scale $\mu^{2}=\left(E_{T}^{* \text { jet1 }}\right)^{2}+Q^{2} / 4$ \\
\hline
\end{tabular}

Table 7. Ratio of integrated $e^{+} p$ diffractive dijet cross sections for $Q^{2}<2 \mathrm{GeV}^{2}$ (photoproduction) to $Q^{2}>4 \mathrm{GeV}^{2}$ (DIS). Listed are the ratios for data and for the NLO calculation including two variants. The data and NLO uncertainties are indicated. The double-ratio of data to NLO and its uncertainties are also given.

\section{Summary}

Diffractive dijet production is measured in photoproduction and deep-inelastic scattering in the same kinematic range $0.2<y<0.7$ and $0.010<x_{\mathbb{P}}<0.024$ for jets with $E_{T}^{* j e t 1}>$ $5.5 \mathrm{GeV}, E_{T}^{* \text { jet2 }}>4.0 \mathrm{GeV}$ and with limits on the photon virtuality $Q^{2}<2 \mathrm{GeV}^{2}$ for photoproduction and $4 \mathrm{GeV}^{2}<Q^{2}<80 \mathrm{GeV}^{2}$ for DIS. For the leading proton detection, the H1 Very Forward Proton Spectrometer is used for the first time, such that the diffractive sample is free of background from low-mass proton dissociative states.

In DIS, diffractive dijet production is well described within the experimental and theoretical uncertainties by the NLO calculations based on the H12006 Fit-B diffractive parton densities of the proton. Within uncertainties, the QCD factorisation assumptions made for the NLO calculation are confirmed in this process. This result is consistent with previous $\mathrm{H} 1$ and ZEUS measurements and the new data may be used in future DPDF fits.

In photoproduction, next-to-leading order (NLO) calculations based on the H12006 Fit-B diffractive parton densities overestimate the measured total cross sections, thus confirming previous H1 measurements, where the Large Rapidity Gap method for the identification of diffractive events was used. The shapes of the differential cross sections are described within the experimental and theoretical uncertainties. There is no hint of dependence of the observed suppression on the variable $x_{\gamma}^{\text {obs }}$.

In order to profit from cancellations of theoretical uncertainties, ratios of photoproduction to DIS cross sections and double ratios of data to NLO are analysed. Integrated over the analysis phase space the double ratio is found to be $0.51 \pm 0.09$. Following this, within the theoretical framework based on diffractive parton densities, factorisation is broken in diffractive dijet photoproduction. This observation is in agreement with previous H1 measurements, where complementary experimental methods have been used. Contributions from proton dissociative processes present in the previous analyses but absent here are ruled out as a cause of the observed suppression. The differential measurements of cross sections and cross section ratios in DIS and photoproduction provide stringent tests of the theory both in normalisation and in shape. 


\begin{tabular}{|c|c|c|c|c|c|c|c|}
\hline$\left|\Delta \eta^{\text {jets }}\right|$ & bin & $\sigma_{\gamma p} / \sigma_{D I S}$ & $\begin{array}{c}\delta_{\text {stat }} \\
{[\%]}\end{array}$ & $\begin{array}{c}\rho_{\text {stat }} \\
{[\%]}\end{array}$ & $\begin{array}{c}\delta_{\text {syst }} \\
{[\%]}\end{array}$ & $\begin{array}{c}\rho_{\text {syst }} \\
{[\%]}\end{array}$ & $1+\delta_{\text {hadr }}$ \\
\hline $0 \div 0.5$ & 1 & 6.9 & 14.9 & & 14.8 & & 0.867 \\
\hline $0.5 \div 1$ & 2 & 9.2 & 21.3 & $\rho_{12}=-10$ & 18.5 & $\rho_{12}=98$ & 0.865 \\
\hline $1 \div 1.5$ & 3 & 7.1 & 24.6 & $\rho_{13}=9 \rho_{23}=-12$ & 22.7 & $\rho_{13}=95 \rho_{23}=97$ & 0.908 \\
\hline $1.5 \div 2$ & 4 & 5.2 & 38.5 & $\rho_{14}=5 \rho_{24}=10 \rho_{34}=-8$ & 33.5 & $\rho_{14}=88 \rho_{24}=90 \rho_{34}=95$ & 0.951 \\
\hline$y$ & bin & $\sigma_{\gamma p} / \sigma_{D I S}$ & $\begin{array}{c}\delta_{\text {stat }} \\
{[\%]}\end{array}$ & $\begin{array}{c}\rho_{\text {stat }} \\
{[\%]}\end{array}$ & $\begin{array}{c}\delta_{\text {syst }} \\
{[\%]}\end{array}$ & $\begin{array}{c}\rho_{\text {syst }} \\
{[\%]}\end{array}$ & $1+\delta_{\text {hadr }}$ \\
\hline $0.2 \div 0.32$ & 1 & 8.0 & 22.7 & & 22.5 & & 0.864 \\
\hline $0.32 \div 0.44$ & 2 & 7.7 & 21.3 & $\rho_{12}=-12$ & 18.4 & $\rho_{12}=87$ & 0.912 \\
\hline $0.44 \div 0.56$ & 3 & 6.2 & 24.1 & $\rho_{13}=12 \rho_{23}=-29$ & 21.5 & $\rho_{13}=88 \rho_{23}=91$ & 0.907 \\
\hline $0.56 \div 0.7$ & 4 & 8.9 & 28.0 & $\rho_{14}=1 \rho_{24}=16 \rho_{34}=-33$ & 14.7 & $\rho_{14}=84 \rho_{24}=84 \rho_{34}=75$ & 0.841 \\
\hline$z_{\mathbb{P}}$ & bin & $\sigma_{\gamma p} / \sigma_{D I S}$ & $\begin{array}{c}\delta_{\text {stat }} \\
{[\%]}\end{array}$ & $\begin{array}{c}\rho_{\text {stat }} \\
{[\%]}\end{array}$ & $\begin{array}{c}\delta_{\text {syst }} \\
{[\%]}\end{array}$ & $\begin{array}{c}\rho_{\text {syst }} \\
{[\%]}\end{array}$ & $1+\delta_{\text {hadr }}$ \\
\hline $0 \div 0.2$ & 1 & 2.29 & 42.7 & & 53.5 & & 0.697 \\
\hline $0.2 \div 0.4$ & 2 & 6.1 & 20.4 & $\rho_{12}=-18$ & 19.1 & $\rho_{12}=80$ & 0.790 \\
\hline $0.4 \div 0.6$ & 3 & 8.5 & 20.7 & $\rho_{13}=9 \rho_{23}=-26$ & 16.3 & $\rho_{13}=85 \rho_{23}=92$ & 0.932 \\
\hline $0.6 \div 0.8$ & 4 & 21.4 & 43.0 & $\rho_{14}=0 \rho_{24}=8 \rho_{34}=-31$ & 22.7 & $\rho_{14}=81 \rho_{24}=75 \rho_{34}=89$ & 1.116 \\
\hline $\begin{array}{l}E_{T}^{* j e t 1} \\
{[\mathrm{GeV}]}\end{array}$ & bin & $\sigma_{\gamma p} / \sigma_{D I S}$ & $\begin{array}{c}\delta_{\text {stat }} \\
{[\%]}\end{array}$ & $\begin{array}{c}\rho_{\text {stat }} \\
{[\%]}\end{array}$ & $\begin{array}{c}\delta_{\text {syst }} \\
{[\%]}\end{array}$ & $\begin{array}{c}\rho_{\text {syst }} \\
{[\%]}\end{array}$ & $1+\delta_{\text {hadr }}$ \\
\hline $5.5 \div 7$ & 1 & 8.0 & 17.1 & & 20.4 & & 0.872 \\
\hline $7 \div 8.5$ & 2 & 8.05 & 26.7 & $\rho_{12}=-39$ & 12.2 & $\rho_{12}=51$ & 0.957 \\
\hline $8.5 \div 10$ & 3 & 4.4 & 61.6 & $\rho_{13}=13 \rho_{23}=-52$ & 28.3 & $\rho_{13}=70 \rho_{23}=90$ & 0.858 \\
\hline $10 \div 14.5$ & 4 & 2.4 & 78.2 & $\rho_{14}=-4 \rho_{24}=16 \rho_{34}=-55$ & 63.5 & $\rho_{14}=57 \rho_{24}=76 \rho_{34}=87$ & 0.859 \\
\hline
\end{tabular}

Table 8. Ratios of differential diffractive dijet $e p$ cross sections, measured in photoproduction, to measurements in DIS as a function of the variables $\left|\Delta \eta^{\text {jets }}\right|, y, z_{\mathbb{P}}$ and $E_{T}^{* \text { jet1 }}$ in the phase space detailed in table 1 . For each data point, the statistical $\left(\delta_{\text {stat }}\right)$ and systematic $\left(\delta_{\text {syst }}\right)$ uncertainties, the corresponding correlation coefficients $\left(\rho_{\text {stat }}, \rho_{\text {syst }}\right)$ and the hadronisation correction factors $\left(1+\delta_{\text {hadr }}\right)$ applied to the NLO calculations are given. 


\section{Acknowledgments}

We are grateful to the HERA machine group whose outstanding efforts have made this experiment possible. We thank the engineers and technicians for their work in constructing and maintaining the $\mathrm{H} 1$ detector, our funding agencies for financial support, the DESY technical staff for continual assistance and the DESY directorate for support and for the hospitality which they extend to the non DESY members of the collaboration. We would like to give credit to all partners contributing to the EGI computing infrastructure for their support for the H1 Collaboration. We express our gratitude to M. Ryskin for helpful discussions.

Open Access. This article is distributed under the terms of the Creative Commons Attribution License (CC-BY 4.0), which permits any use, distribution and reproduction in any medium, provided the original author(s) and source are credited.

\section{References}

[1] P. Van Esch et al., The $H 1$ forward proton spectrometer at HERA, Nucl. Instrum. Meth. A 446 (2000) 409 [hep-ex/0001046] [INSPIRE].

[2] A. Astvatsatourov et al., The H1 very forward proton spectrometer at HERA, Nucl. Instrum. Meth. A 736 (2014) 46 [INSPIRE].

[3] J.C. Collins, Proof of factorization for diffractive hard scattering, Phys. Rev. D 57 (1998) 3051 [Erratum ibid. D 61 (2000) 019902] [hep-ph/9709499] [INSPIRE].

[4] H1 collaboration, A. Aktas et al., Measurement and QCD analysis of the diffractive deep-inelastic scattering cross-section at HERA, Eur. Phys. J. C 48 (2006) 715 [hep-ex/0606004] [INSPIRE].

[5] ZEUS collaboration, S. Chekanov et al., Dissociation of virtual photons in events with a leading proton at HERA, Eur. Phys. J. C 38 (2004) 43 [hep-ex/0408009] [INSPIRE].

[6] ZEUS collaboration, S. Chekanov et al., Measurement of diffractive production of $D^{* \pm}(2010)$ mesons in deep inelastic scattering at HERA, Phys. Lett. B 545 (2002) 244 [hep-ex/0206020] [INSPIRE].

[7] H1 collaboration, A. Aktas et al., Diffractive open charm production in deep-inelastic scattering and photoproduction at HERA, Eur. Phys. J. C 50 (2007) 1 [hep-ex/0610076] [INSPIRE].

[8] H1 collaboration, A. Aktas et al., Dijet cross sections and parton densities in diffractive DIS at HERA, JHEP 10 (2007) 042 [arXiv:0708.3217] [INSPIRE].

[9] ZEUS collaboration, S. Chekanov et al., Dijet production in diffractive deep inelastic scattering at HERA, Eur. Phys. J. C 52 (2007) 813 [arXiv:0708.1415] [InSPIRE].

[10] H1 collaboration, F.D. Aaron et al., Measurement of dijet production in diffractive deep-inelastic scattering with a leading proton at HERA, Eur. Phys. J. C 72 (2012) 1970 [arXiv: 1111.0584] [INSPIRE].

[11] H1 collaboration, V. Andreev et al., Measurement of dijet production in diffractive deep-inelastic ep scattering at HERA, JHEP 03 (2015) 092 [arXiv: 1412.0928] [INSPIRE]. 
[12] CDF collaboration, T. Affolder et al., Diffractive dijets with a leading antiproton in $\bar{p} p$ collisions at $\sqrt{s}=1800$ GeV, Phys. Rev. Lett. 84 (2000) 5043 [InSPIRE].

[13] CMS collaboration, Observation of a diffractive contribution to dijet production in proton-proton collisions at $\sqrt{s}=7$ TeV, Phys. Rev. D 87 (2013) 012006 [arXiv:1209.1805] [INSPIRE].

[14] A.B. Kaidalov, V.A. Khoze, A.D. Martin and M.G. Ryskin, Probabilities of rapidity gaps in high-energy interactions, Eur. Phys. J. C 21 (2001) 521 [hep-ph/0105145] [INSPIRE].

[15] A.B. Kaidalov, V.A. Khoze, A.D. Martin and M.G. Ryskin, Factorization breaking in diffractive dijet production, Phys. Lett. B 559 (2003) 235 [hep-ph/0302091] [INSPIRE].

[16] J.C. Collins, Factorization in hard diffraction, J. Phys. G 28 (2002) 1069 [hep-ph/0107252] [INSPIRE].

[17] H1 collaboration, F.D. Aaron et al., Diffractive dijet photoproduction in ep collisions at HERA, Eur. Phys. J. C 70 (2010) 15 [arXiv:1006.0946] [InSPIRE].

[18] H1 collaboration, A. Aktas et al., Tests of QCD factorisation in the diffractive production of dijets in deep-inelastic scattering and photoproduction at HERA,

Eur. Phys. J. C 51 (2007) 549 [hep-ex/0703022] [InSPIRE].

[19] ZEUS collaboration, S. Chekanov et al., Diffractive photoproduction of dijets in ep collisions at HERA, Eur. Phys. J. C 55 (2008) 177 [arXiv:0710.1498] [InSPIRE].

[20] ZEUS collaboration, S. Chekanov et al., A QCD analysis of ZEUS diffractive data, Nucl. Phys. B 831 (2010) 1 [arXiv:0911.4119] [INSPIRE].

[21] A.B. Kaidalov, V.A. Khoze, A.D. Martin and M.G. Ryskin, Unitarity effects in hard diffraction at HERA, Phys. Lett. B 567 (2003) 61 [hep-ph/0306134] [INSPIRE].

[22] A.B. Kaidalov, V.A. Khoze, A.D. Martin and M.G. Ryskin, Factorization breaking in diffractive dijet photoproduction at HERA, Eur. Phys. J. C 66 (2010) 373 [arXiv:0911.3716] [INSPIRE].

[23] C.F. von Weizsäcker, Radiation emitted in collisions of very fast electrons, Z. Phys. 88 (1934) 612 [inSPIRE].

[24] E.J. Williams, Nature of the high-energy particles of penetrating radiation and status of ionization and radiation formulae, Phys. Rev. 45 (1934) 729 [INSPIRE].

[25] M. Glück, E. Reya and A. Vogt, Parton structure of the photon beyond the leading order, Phys. Rev. D 45 (1992) 3986 [INSPIRE].

[26] P. Aurenche, M. Fontannaz and J.P. Guillet, New NLO parametrizations of the parton distributions in real photons, Eur. Phys. J. C 44 (2005) 395 [hep-ph/0503259] [INSPIRE].

[27] G. Ingelman and P.E. Schlein, Jet structure in high mass diffractive scattering, Phys. Lett. B 152 (1985) 256 [INSPIRE].

[28] T. Regge, Introduction to complex orbital momenta, Nuovo Cim. 14 (1959) 951 [INSPIRE].

[29] S. Frixione, Z. Kunszt and A. Signer, Three jet cross-sections to next-to-leading order, Nucl. Phys. B 467 (1996) 399 [hep-ph/9512328] [INSPIRE].

[30] Z. Nagy and Z. Trócsányi, Next-to-leading order calculation of four jet observables in electron positron annihilation, Phys. Rev. D 59 (1999) 014020 [Erratum ibid. D 62 (2000) 099902] [hep-ph/9806317] [INSPIRE]. 
[31] Z. Nagy and Z. Trócsányi, Multijet cross-sections in deep inelastic scattering at next-to-leading order, Phys. Rev. Lett. 87 (2001) 082001 [hep-ph/0104315] [INSPIRE].

[32] M. Klasen and G. Kramer, Inclusive two jet production at HERA: direct and resolved cross-sections in next-to-leading order QCD, Z. Phys. C 76 (1997) 67 [hep-ph/9611450] [INSPIRE].

[33] M. Klasen and G. Kramer, Review of factorization breaking in diffractive photoproduction of dijets, Mod. Phys. Lett. A 23 (2008) 1885 [arXiv:0806.2269] [InSPIRE].

[34] K. Černý, Tests of QCD hard factorization in diffractive photoproduction of dijets at HERA, $\mathrm{Ph} . D$. thesis, available at http://www-h1.desy.de/publications/theses_list.html, Charles University in Prague, Prague Czech Republic (2008) [INSPIRE].

[35] J. Chýla, J. Cvach, K. Sedlák and M. Taševský, QCD analysis of dijet production at low boldmath $Q^{2}$ at HERA, Eur. Phys. J. C 40 (2005) 469 [hep-ph/0501065] [InSPIRE].

[36] H1 collaboration, I. Abt et al., The H1 detector at HERA, Nucl. Instrum. Meth. A 386 (1997) 310 [INSPIRE].

[37] H1 collaboration, I. Abt et al., The tracking, calorimeter and muon detectors of the H1 experiment at HERA, Nucl. Instrum. Meth. A 386 (1997) 348 [INSPIRE].

[38] H1 SPACAL Group collaboration, R.D. Appuhn et al., The H1 lead/scintillating fiber calorimeter, Nucl. Instrum. Meth. A 386 (1997) 397 [INSPIRE].

[39] D. Pitzl et al., The H1 silicon vertex detector, Nucl. Instrum. Meth. A 454 (2000) 334 [hep-ex/0002044] [INSPIRE].

[40] J. Becker et al., A vertex trigger based on cylindrical multiwire proportional chambers, Nucl. Instrum. Meth. A 586 (2008) 190 [physics/0701002] [INSPIRE].

[41] H1 Calorimeter Group collaboration, B. Andrieu et al., The $H 1$ liquid argon calorimeter system, Nucl. Instrum. Meth. A 336 (1993) 460 [INSPIRE].

[42] H1 Calorimeter Group collaboration, B. Andrieu et al., Beam tests and calibration of the H1 liquid argon calorimeter with electrons, Nucl. Instrum. Meth. A 350 (1994) 57 [INSPIRE].

[43] H1 Calorimeter Group collaboration, B. Andrieu et al., Results from pion calibration runs for the $H 1$ liquid argon calorimeter and comparisons with simulations, Nucl. Instrum. Meth. A 336 (1993) 499 [INSPIRE].

[44] D. Šalek, Measurement of the longitudinal proton structure function in diffraction at the $H 1$ experiment and prospects for diffraction at LHC, Ph.D. thesis, also available at http://www-h1.desy.de/publications/theses_list.html, Charles University in Prague, Prague Czech Republic (2011) [DESY-THESIS-2011-013] [INSPIRE].

[45] H1 collaboration, F.D. Aaron et al., Determination of the integrated luminosity at HERA using elastic QED Compton events, Eur. Phys. J. C 72 (2012) 2163 [arXiv:1205.2448] [INSPIRE].

[46] T. Hreus, Studies of diffractive scattering of photons at large momentum transfer and of the VFPS detector at HERA, Ph.D. thesis, also available at http://www-h1.desy.de/publications/theses_list.html, Université Libre de Bruxelles, Bruxelles Belgium (2008) [DESY-THESIS-2008-040] [INSPIRE]. 
[47] M. Peez, Search for deviations from the standard model in high transverse energy processes at the electron proton collider HERA, Ph.D. thesis, also available at http://www-h1.desy.de/publications/theses_list.html, Université Claude Bernard — Lyon 1, Lyon France (2003) [DESY-THESIS-2003-023] [INSPIRE].

[48] S. Hellwig, Untersuchung der $D^{*}$ - $\pi_{\text {slow }}$ Double Tagging Methode in Charmanalysen (in German), Master's thesis, also available at http://www-h1.desy.de/publications/theses_list.html, University of Hamburg, Hamburg Germany (2004) [INSPIRE].

[49] S. Catani, Y.L. Dokshitzer and B.R. Webber, The $K^{-}$perpendicular clustering algorithm for jets in deep inelastic scattering and hadron collisions, Phys. Lett. B 285 (1992) 291 [INSPIRE].

[50] M. Cacciari, G.P. Salam and G. Soyez, FastJet user manual, Eur. Phys. J. C 72 (2012) 1896 [arXiv:1111.6097] [INSPIRE].

[51] H1 collaboration, C. Adloff et al., Inclusive measurement of diffractive deep inelastic ep scattering, Z. Phys. C 76 (1997) 613 [hep-ex/9708016] [INSPIRE].

[52] J. Delvax, Etude de la production de jets en diffraction à HERA, à l'aide du spectromètre à protons VFPS (in French), Ph.D. thesis, also available at http://www-h1.desy.de/publications/theses_list.html, Université Libre de Bruxelles, Bruxelles Belgium (2010) [DESY-THESIS-2010-045] [INSPIRE].

[53] A. Glazov, N. Raicevic and A. Zhokin, Fast simulation of showers in the H1 calorimeter, Comput. Phys. Commun. 181 (2010) 1008 [InSPIRE].

[54] M. Klasen and G. Kramer, Dijet cross-sections at $O\left(\alpha_{s}^{2}\right)$ in photon-proton collisions, Phys. Lett. B 366 (1996) 385 [hep-ph/9508337] [INSPIRE].

[55] S. Frixione and G. Ridolfi, Jet photoproduction at HERA, Nucl. Phys. B 507 (1997) 315 [hep-ph/9707345] [INSPIRE].

[56] R. Brun, F. Bruyant, M. Maire, A. McPherson and P. Zanarini, GEANT detector description and simulation tool, CERN Program Library Long Writeup W5013, CERN, Geneva Switzerland (1987).

[57] H. Jung, Hard diffractive scattering in high-energy ep collisions and the Monte Carlo generator RAPGAP, Comput. Phys. Commun. 86 (1995) 147 [INSPIRE].

[58] B. Andersson, G. Gustafson, G. Ingelman and T. Sjöstrand, Parton fragmentation and string dynamics, Phys. Rept. 97 (1983) 31 [INSPIRE].

[59] T. Sjöstrand, PYTHIA 5.7 and JETSET 7.4: physics and manual, hep-ph/9508391 [INSPIRE].

[60] F.D. Aaron et al., Measurement of the cross section for diffractive deep-inelastic scattering with a leading proton at HERA, Eur. Phys. J. C 71 (2011) 1578 [arXiv:1010.1476] [INSPIRE].

[61] S. Schmitt, TUnfold: an algorithm for correcting migration effects in high energy physics, 2012 JINST 7 T10003 [arXiv:1205.6201] [INSPIRE].

[62] A. Tikhonov, Solution of incorrectly formulated problems and the regularization method, Sov. Math. 4 (1963) 1035. 
[63] H1 collaboration, V. Andreev et al., Measurement of multijet production in ep collisions at high $Q^{2}$ and determination of the strong coupling $\alpha_{s}$, Eur. Phys. J. C 75 (2015) 65 [arXiv: 1406.4709] [INSPIRE].

[64] W. Schütte, M. Wendt and K. Mess, The new directional coupler pick up for the HERA proton beam position monitoring system, in Proceedings of "1987 IEEE Particle Accelerator Conference", E.R. Lindstrom and L.S. Taylor eds., Washington U.S.A. (1987) [Conf. Proc. C 870316 (1987) 1725] [INSPIRE].

[65] H1 collaboration, A. Aktas et al., Measurement of dijet production at low $Q^{2}$ at HERA, Eur. Phys. J. C 37 (2004) 141 [hep-ex/0401010] [InSPIRE].

[66] K.J. Golec-Biernat and A. Luszczak, Diffractive parton distributions from the analysis with higher twist, Phys. Rev. D 76 (2007) 114014 [arXiv:0704.1608] [INSPIRE]. 